\title{
A MIRROR THEOREM FOR THE MIRROR QUINTIC
}

\author{
Y.-P. LEE AND M. SHOEMAKER
}

\begin{abstract}
The celebrated Mirror Theorem states that the genus zero part of the $A$ model (quantum cohomology, rational curves counting) of the Fermat quintic threefold is equivalent to the $B$ model (complex deformation, variation of Hodge structure) of its mirror dual orbifold. In this article, we establish a mirror-dual statement. Namely, the $B$ model of the Fermat quintic threefold is shown to be equivalent to the $A$ model of its mirror, and hence establishes the mirror symmetry as a true duality.
\end{abstract}

\section{CONTENTS}

0. Introduction

1. Quantum orbifold cohomology

2. J-function of $\left[\mathbb{P}^{4} / \bar{G}\right]$

3. A model of the mirror quintic $\mathcal{W}$

4. Periods and Picard-Fuchs equations 29

5. $B$ model of the Fermat quintic $M \quad 32$

6. Mirror Theorem for the mirror quintic: $A(\mathcal{W}) \equiv B(M)$

References

\section{INTRODUCTION}

0.1. Mirror Theorem for the Fermat quintic threefold. Let $M$ be the Fermat quintic threefold defined by

$$
M:=\left\{x_{0}^{5}+x_{1}^{5}+x_{3}^{5}+x_{4}^{5}+x_{5}^{5}=0\right\} \subset \mathbb{P}^{4} .
$$

The Greene-Plesser [19] mirror construction gives the mirror orbifold as the quotient stack

$$
\mathcal{W}:=[M / \bar{G}],
$$

where $\bar{G} \cong(\mathbb{Z} / 5 \mathbb{Z})^{3}$ is a (finite abelian) subgroup of the big torus of $\mathbb{P}^{4}$ acting via generators $e_{1}, e_{2}, e_{3}$ :

$$
\begin{aligned}
& e_{1}\left[x_{0}, x_{1}, x_{2}, x_{3}, x_{4}\right]=\left[\zeta x_{0}, x_{1}, x_{2}, x_{3}, \zeta^{-1} x_{4}\right] \\
& e_{2}\left[x_{0}, x_{1}, x_{2}, x_{3}, x_{4}\right]=\left[x_{0}, \zeta x_{1}, x_{2}, x_{3}, \zeta^{-1} x_{4}\right] \\
& e_{3}\left[x_{0}, x_{1}, x_{2}, x_{3}, x_{4}\right]=\left[x_{0}, x_{1}, \zeta x_{2}, x_{3}, \zeta^{-1} x_{4}\right] .
\end{aligned}
$$

Assuming the validity of mirror symmetry for the mirror pair $(M, \mathcal{W})$, Candelas-de la Ossa-Green-Parkes made the celebrated calculation which 
in particular predicted the number of rational curves in the Fermat quintic of any degree. This calculation was verified in full generality only after many years of works, involving many distinguished mathematicians and culminating in the proof by A. Givental [13] (and Liu-Lian-Yau [17]). The mathematical proof of the CDGP Conjecture was termed the Mirror Theorem for the Fermat quintic threefold.

In a way, what the Mirror Theorem says is that the invariants from the complex deformations of $\mathcal{W}$ matches those from the Kähler deformations of $M$, up to a change of variables termed the mirror map. In terms of E. Witten's terminology [20], the above mirror theorem states that the (genus 0) $A$ model of $M$ is equivalent to $B$ model of $\mathcal{W}$. This can be formulated in mathematical terms as saying that the genus zero Gromov-Witten theory (GWT), or quantum cohomology, on $M$ is equal to the variation of Hodge structures (VHS) associated to the complex deformations of $\mathcal{W}$.

The complex deformation of Calabi-Yau's is unobstructed by BogomolovTian-Todorov. The dimension of the Kodaira-Spencer space can be identified as the Hodge number $h^{2,1}$ due to the Calabi-Yau property $K \cong \mathscr{O}$. In this case $h^{2,1}(\mathcal{W})=1$. CDGP chose the following one-dimensional deformation family $\left\{\mathcal{W}_{\psi}\right\}=\left\{Q_{\psi}(x)=0\right\}$, where

$$
Q_{\psi}(x)=x_{0}^{5}+x_{1}^{5}+x_{3}^{5}+x_{4}^{5}+x_{5}^{5}-\psi x_{0} x_{1} x_{2} x_{3} x_{4} x_{5}
$$

of hypersurfaces in $\left[\mathbb{P}^{4} / \bar{G}\right]$, such that $\psi=\infty$ is the maximally degenerate moduli point. We note that it is often convenient to use $t=-5 \log \psi$ as the variable. By local Torelli for Calabi-Yau, the deformation is embedded into VHS, which then gives all information about the complex deformation.

The Kähler deformation is given by genus zero GWT along the "small" variable $t$, which is the dual coordinate for the hyperplane class $H . H^{1,1}(M)_{\mathbb{C}}$ is often called the complexified Kähler moduli.

We can rephrase the above in much more precise terms. Both genus zero GWT and VHS can be described by differential systems associated to flat connections. For GWT, it is the Dubrovin connection; for VHS the GaussManin connection. The definitions can be found in Sections 1 and 4 respectively. Therefore, we can phrase the Mirror Theorem for the Fermat quintic in the following form.

Theorem 0.1 (= Theorem 6.6). The fundamental solutions of the Gauss-Manin connection for $\mathcal{W}_{t}$ are equivalent, up to a mirror map, to the fundamental solutions of the Dubrovin connection for $M$, when restricted to $H^{2}(M)$.

0.2. Mirror Theorem for the mirror quintic. Theorem 0.1 can be stated suggestively as

$$
A \text { model of } M \equiv B \text { model of } \mathcal{W} \text {. }
$$

In order for the mirror symmetry to be a true duality, one will also have to show that

$$
B \text { model of } M \equiv A \text { model of } \mathcal{W} \text {. }
$$


This is the task we set for ourselves in this paper.

The first thing we note is that $\mathcal{W}$ is an orbifold. Thus we must replace the singular cohomology by the Chen-Ruan cohomology, and the usual Gromov-Witten theory by the orbifold GWT. These are defined in Section 1 .

Upon a closer look, however, there is a serious technical issue. In the B model of $M$, the Kodaira-Spencer space is of dimension 101 and the VHS of $H^{3}(M)$ is a system of rank 204, thus a calculation of the full Gauss-Manin connection for $M$ is unfeasible. As a first step however, we choose a onedimensional deformation family $\left\{M_{t}\right\}$ defined by the vanishing of (0.1.1), reinterpreted as a family in $\mathbb{P}^{4}$. Similarly, in the $A$ model of $\mathcal{W}$, we have $h_{C R}^{1,1}(\mathcal{W})=101$, where the subscript denotes Chen-Ruan cohomology. We choose the one-dimensional subspace of the complexified Kähler moduli spanned by the hyperplane class and call the coordinate $t$ as before. These one dimensional families are arguably the most natural and the most important dimension.

With these choices, the Gauss-Manin system for $M$ still has rank 204, but over a one dimensional base. The fundamental solution is a matrix of size 204 by 204 in one variable. The Dubrovin connection on $H_{C R}^{\text {even }}(\mathcal{W})$ likewise has the fundamental solution matrix of size 204 by 204. Here $204=$ $\operatorname{dim} H_{C R}^{\text {even }}(\mathcal{W})$.

The main result of this paper is the following theorem.

Theorem 0.2 (= Theorem 6.8). The fundamental solutions of the Gauss-Manin connection for $\left\{M_{t}\right\}$ are equivalent, up to a mirror map, to the fundamental solutions of the Dubrovin connection for $\mathcal{W}$ restricted to $t \in H^{2}(\mathcal{W})$.

0.3. Outline of the paper. We have in mind the readership with diverse background. For convenience, we have included short introductions in Section 1 and Section 4 to orbifold Gromov-Witten theory and the theory of variation of Hodge structures, recalling only facts pertinent to our presentation. Sections 2 and 3 present the $A$ model calculation for $\mathcal{W}$. We first calculate the genus zero Gromov-Witten theory for $\left[\mathbb{P}^{4} / \bar{G}\right]$ in Section 2 , we then calculate the genus zero Gromov-Witten theory for $\mathcal{W}$ in Section 3 . In Section 5 we present a reformulation of the results from [12], and summarize our $B$ model calculation for $M_{t}$. In the last section, we prove our main result, showing the validity of the Mirror-dual statement of the Mirror Theorem. For the benefit of our dual readership, we include a derivation of Theorem 0.1 from the usual statement of the Mirror Theorem.

Acknowledgements. Y.P.L. would like to thank his collaborators Profs. H.W. Lin and C.-L. Wang. In particular, he learns most of what little he knows about the Hodge theory from his collaborative projects with them. Y.P.L. is partially supported by the NSF.

M.S. would like to thank his advisor, Prof. Y. Ruan for his help and guidance over the years, and for first introducing him to this beautiful subject. 
He is also grateful to Prof. R. Cavalieri for many useful conversations. M.S. was partially supported by NSF RTG grant DMS-0602191.

\section{QUANTUM ORBIFOLD COHOMOLOGY}

In this section we give a brief review of Chen-Ruan cohomology and quantum orbifold cohomology, with the parallel goal of setting notation. A more detailed general review can be found in [8].

Conventions 1.1. We work in the algebraic category. The term orbifold means "smooth separated Deligne-Mumford stack of finite type over $\mathbb{C}$."

The various dimensions are complex dimensions. On the other hand, the degrees of cohomology are all in real/topological degrees.

Unless otherwise stated all cohomology groups have coefficients in $\mathbb{C}$.

1.1. Chen-Ruan cohomology groups. Let $\mathcal{X}$ be a stack. Its inertia stack $I \mathcal{X}$ is the fiber product

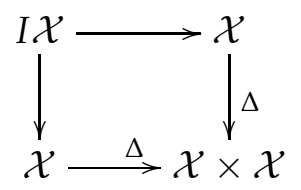

where $\Delta$ is the diagonal map. The fiber product is taken in the 2-category of stacks. One can think of a point of $I \mathcal{X}$ as a pair $(x, g)$ where $x$ is a point of $\mathcal{X}$ and $g \in \operatorname{Aut}_{\mathcal{X}}(x)$. There is an involution $I: I \mathcal{X} \rightarrow I \mathcal{X}$ which sends the point $(x, g)$ to $\left(x, g^{-1}\right)$. It is often convenient to call the components of $I \mathcal{X}$ for which $g \neq e$ the twisted sectors.

If $\mathcal{X}=[V / G]$ is a global quotient of a nonsingular variety $V$ by a finite group $G, I \mathcal{X}$ takes a particularly simple form. Let $S_{G}$ denote the set of conjugacy classes $(g)$ in $G$, then

$$
I[V / G]=\coprod_{(g) \in S_{G}}\left[V^{g} / C(g)\right] .
$$

The Chen-Ruan orbifold cohomology groups $H_{C R}^{*}(X)([5])$ of a Deligne-Mumford stack $\mathcal{X}$ are the cohomology groups of its inertia stack

$$
H_{C R}^{*}(\mathcal{X}):=H^{*}(I \mathcal{X}) .
$$

Let $(x, g)$ be a geometric point in a component $\mathcal{X}_{i}$ of $I \mathcal{X}$. By definition $g \in \operatorname{Aut}_{\mathcal{X}}(x)$. Let $r$ be the order of $g$. Then the $g$-action on $T_{x} \mathcal{X}$ decomposes as eigenspaces

$$
T_{x} \mathcal{X}=\bigoplus_{0 \leq j<r} E_{j}
$$

where $E_{j}$ is the subspace of $T_{x} \mathcal{X}$ on which $g$ acts by multiplication by $\exp (2 \pi \sqrt{-1} j / r)$. Define the age of $\mathcal{X}_{i}$ to be

$$
\operatorname{age}\left(\mathcal{X}_{i}\right):=\sum_{j=0}^{r-1} \frac{j}{r} \operatorname{dim}\left(E_{j}\right)
$$


This is independent of the choice of geometric point $(x, g) \in \mathcal{X}_{i}$.

Let $\alpha$ be an element in $H^{p}\left(\mathcal{X}_{i}\right) \subset H^{*}(I \mathcal{X})$. Define the age-shifted degree of $\alpha$ to be

$$
\operatorname{deg}_{C R}(\alpha):=p+2 \operatorname{age}\left(\mathcal{X}_{i}\right)
$$

This defines a grading on $H_{C R}(\mathcal{X})$.

When $\mathcal{X}$ is compact the orbifold Poincaré pairing is defined by

$$
\left(\alpha_{1}, \alpha_{2}\right)_{C R}^{\mathcal{X}}:=\int_{I \mathcal{X}} \alpha_{1} \cup I^{*}\left(\alpha_{2}\right),
$$

where $\alpha_{1}$ and $\alpha_{2}$ are elements of $H_{C R}^{*}(\mathcal{X})$. It is easy to see that when $\alpha_{1}$ and $\alpha_{2}$ are homogeneous elements, $\left(\alpha_{1}, \alpha_{2}\right)_{C R} \neq 0$ only if $\operatorname{deg}_{C R}\left(\alpha_{1}\right)+$ $\operatorname{deg}_{C R}\left(\alpha_{2}\right)=2 \operatorname{dim}(\mathcal{X})$.

\subsection{Orbifold Gromov-Witten theory.}

1.2.1. Orbifold Gromov-Witten invariants. We follow the standard references [6] and [1] of orbifold Gromov-Witten theory.

Given an orbifold $\mathcal{X}$, there exists a moduli space $\overline{\mathscr{M}}_{g, n}(\mathcal{X}, d)$ of stable maps from $n$-marked genus $g$ pre-stable orbifold curves to $\mathcal{X}$ of degree $d \in H_{2}(\mathcal{X} ; \mathbb{Q})$. Each source curve $\left(\mathcal{C}, p_{1}, \ldots, p_{n}\right)$ has non-trivial orbifold structure only at the nodes and marked points: At each (orbifold) marked point it is a cyclic quotient stack and at each node a balanced cyclic quotient. That is, étale locally isomorphic to

$$
\left[\operatorname{Spec}\left(\frac{\mathbb{C}[x, y]}{(x y)}\right) / \mu_{r}\right],
$$

where $\zeta \in \mu_{r}$ acts as $(x, y) \mapsto\left(\zeta x, \zeta^{-1} y\right)$. The maps are required to be representable at each node.

Each marked point $p_{i}$ is étale locally isomorphic to $\left[\mathbb{C} / \mu_{r_{i}}\right]$. There is an induced homomorphism

$$
\mu_{r_{i}} \rightarrow \operatorname{Aut}_{\mathcal{X}}\left(f\left(p_{i}\right)\right)
$$

Maps in $\overline{\mathscr{M}}_{g, n}(\mathcal{X}, d)$ are required be representable, which amounts to saying that these homomorphisms be injective. For each marked point $p_{i}$, one can thus associate a point $\left(x_{i}, g_{i}\right)$ in $I \mathcal{X}$ where $x_{i}=f\left(p_{i}\right)$, and $g_{i} \in \operatorname{Aut}_{\mathcal{X}}\left(x_{i}\right)$ is the image of $\exp \left(2 \pi \sqrt{-1} / r_{i}\right)$ under the induced homomorphism.

Given a family $\mathcal{C} \rightarrow S$ of marked orbifold curves, there may be nontrivial gerbe structure above the locus defined by the $i$-th marked point. For this reason there is generally not a well defined map

$$
e v_{i}: \overline{\mathscr{M}}_{g, n}(\mathcal{X}, d) \rightarrow I \mathcal{X}
$$

However, as explained in [1] and [8] Section 2.2.2, it is still possible to define maps

$$
e v_{i}^{*}: H_{C R}^{*}(\mathcal{X}) \rightarrow H^{*}\left(\overline{\mathscr{M}}_{g, n}(\mathcal{X}, d)\right)
$$

which behave as if the evaluation maps $e v_{i}$ are well defined. 
Let $X$ denote the coarse underlying space of the stack $\mathcal{X}$. There is a reification map

$$
\overline{\mathscr{M}}_{g, n}(\mathcal{X}, d) \rightarrow \overline{\mathscr{M}}_{g, n}(X, d),
$$

which forgets the orbifold structure of each map. For each marked point there is an associated line bundle, the $i^{\text {th }}$ universal cotangent line bundle,

$$
\begin{gathered}
L_{i} \\
\downarrow \\
\overline{\mathscr{M}}_{g, n}(X, d)
\end{gathered}
$$

with fiber $T_{p_{i}}^{*} C$ over $\left\{f:\left(C, p_{1}, \ldots, p_{n}\right) \rightarrow X\right\}$. Define the $i$-th $\psi$-class by $\psi_{i}=r^{*}\left(c_{1}\left(L_{i}\right)\right)$.

As in the non-orbifold setting, there exists a virtual fundamental class $\left[\overline{\mathscr{M}}_{g, n}(\mathcal{X}, d)\right]^{\text {vir }}$. Orbifold Gromov-Witten invariants for $\mathcal{X}$ are defined as integrals

$$
\left\langle\alpha_{1} \psi^{k_{1}}, \ldots, \alpha_{n} \psi^{k_{n}}\right\rangle_{g, n, d}^{\mathcal{X}}=\int_{\left[\overline{\mathscr{M}}_{g, n}(\mathcal{X}, d)\right]^{v i r}} \prod_{i=1}^{n} e v_{i}^{*}\left(\alpha_{i}\right) \psi_{i}^{k_{i}}
$$

where $\alpha_{i} \in H_{C R}^{*}(\mathcal{X})$.

Let $\overline{\mathscr{M}}_{g,\left(g_{1}, \ldots, g_{n}\right)}(\mathcal{X}, d)$ denote the open and closed substack of $\overline{\mathscr{M}}_{g, n}(\mathcal{X}, d)$ such that $e v_{i}$ maps to a component $\mathcal{X}_{g_{i}}$ of $I \mathcal{X}$. The space $\overline{\mathscr{M}}_{g_{1}\left(g_{1}, \ldots, g_{n}\right)}(\mathcal{X}, d)$ has (complex) virtual dimension

$$
n+(g-1)(\operatorname{dim} \mathcal{X}-3)+\left\langle c_{1}(T \mathcal{X}), d\right\rangle-\sum_{i=0}^{n} \operatorname{age}\left(\mathcal{X}_{g_{i}}\right)
$$

In other words, for homogeneous classes $\alpha_{i} \in H^{*}\left(\mathcal{X}_{g_{i}}\right)$ the Gromov-Witten invariant $\left\langle\alpha_{1}, \ldots, \alpha_{n}\right\rangle_{g, n, d}^{\mathcal{X}}$ will vanish unless

$$
\sum_{i=1}^{n} \operatorname{deg}_{C R}\left(\alpha_{i}\right)=2\left(n+(g-1)(\operatorname{dim} \mathcal{X}-3)+\left\langle c_{1}(T \mathcal{X}), d\right\rangle\right) .
$$

1.2.2. Quantum cohomology and the Dubrovin connection. Let $\left\{T_{i}\right\}_{i \in I}$ be a basis for $H_{C R}^{*}(\mathcal{X})$ and $\left\{T^{i}\right\}_{i \in I}$ its dual basis. We can represent a general point in coordinates by

$$
\mathbf{t}=\sum_{i} t^{i} T_{i} \in H_{C R}^{*}(\mathcal{X})
$$

Gromov-Witten invariants allow us to define a family of product structures parameterized by $\mathbf{t}$ in a formal neighborhood of 0 in $H_{C R}^{*}(\mathcal{X})$. The (big) quantum product $*_{\mathrm{t}}$ is defined as

$$
\alpha_{1} *_{\mathbf{t}} \alpha_{2}:=\sum_{d} \sum_{n \geq 0} \sum_{i} \frac{q^{d}}{n !}\left\langle\alpha_{1}, \alpha_{2}, T_{i}, \mathbf{t}, \ldots, \mathbf{t}\right\rangle_{0,3+n, d}^{\mathcal{X}} T^{i},
$$

where the first sum is over the Mori cone of effective curve classes and the variables $q^{d}$ are in an appropriate Novikov ring $\Lambda$ used to guarantee formal convergence of the sum. The WDVV equations ([9], Section 8.2.3) 
imply the associativity of the product. The small quantum product is defined by restricting the parameter of the quantum product to divisors $\mathbf{t} \in H^{2}(\mathcal{X})$ supported on the non-twisted sector.

One can interpret $*_{\mathbf{t}}$ as defining a product structure on the tangent bundle $T H_{C R}^{*}(\mathcal{X} ; \Lambda)$, such that for a fixed $\mathbf{t}$ the quantum product defines a (Frobenius) algebra structure on $T_{\mathbf{t}} H_{C R}^{*}(\mathcal{X} ; \Lambda)$. This can be rephrased in terms of the Dubrovin connection:

$$
\nabla_{\frac{\partial}{\partial t^{i}}}^{z}\left(\sum_{j} a_{j} T_{j}\right)=\sum_{j} \frac{\partial a_{j}}{\partial t^{i}} T_{j}-\frac{1}{z} \sum_{j} a_{j} T_{i} *_{\mathbf{t}} T_{j} .
$$

This defines a $z$-family of connections on $T H_{C R}^{*}(\mathcal{X} ; \Lambda)$.

Remark 1.2. Note that when $\mathbf{t}, T_{i}$ and $T_{j}$ are in $H_{C R}^{e v e n}(\mathcal{X})$, then for dimension reasons $T_{i} *_{\mathbf{t}} T_{j}$ will be also be supported in even degree. Thus $\nabla^{z}$ restricts to a connection on $T H_{C R}^{\text {even }}(\mathcal{X} ; \Lambda)$. When restricted to $T H_{C R}^{\text {even }}(\mathcal{X} ; \Lambda)$, the quantum product is commutative.

Remark 1.3. For the purpose of this paper, we clarify here what we mean by " $A$ model of $\mathcal{X}$ ". Let $H:=H_{C R}^{\text {even }}(\mathcal{X} ; \Lambda)$. The (genus zero part of) $A$ model of $\mathcal{X}$ is the tangent bundle $T H$ with its natural (flat) fiberwise pairing and the Dubrovin connection restricted to $H_{C R}^{1,1}(\mathcal{X})$.

The commutativity and associativity of the quantum product implies that the Dubrovin connection is flat. The topological recursion relations allow us to explicitly describe solutions to $\nabla^{z}$. Define

$$
s_{i}(\mathbf{t}, z)=T_{i}+\sum_{d} \sum_{n \geq 0} \sum_{j} \frac{q^{d}}{n !}\left\langle\frac{T_{i}}{z-\psi_{1}}, T^{j}, \mathbf{t}, \ldots, \mathbf{t}\right\rangle_{0,2+n, d}^{\mathcal{X}} T_{j}
$$

where $1 /\left(z-\psi_{1}\right)$ should be viewed as a power series in $1 / z$. The sections $s_{i}$ form a basis for the $\nabla^{z}$-flat sections; see e.g. [9], Proposition 10.2.1. Thus we obtain a fundamental solution matrix $S=S(\mathbf{t}, z)=\left(s_{i j}\right)$ given by

$$
s_{i j}(\mathbf{t}, z)=\left(T^{i}, s_{j}\right)_{C R}^{\mathcal{X}}
$$

If one restricts the base to divisors $\mathbf{t} \in H^{2}(\mathcal{X})$, the divisor equation ([1] Theorem 8.3.1) allows a substantial simplification of the formula for $s_{i}$

$$
\left.s_{i}(\mathbf{t}, z)\right|_{\mathbf{t} \in H^{2}(\mathcal{X})}=e^{\mathbf{t} / z}\left(T_{i}+\sum_{d>0} \sum_{j} q^{d} e^{d \mathbf{t}}\left\langle\frac{T_{i}}{z-\psi_{1}}, T^{j}\right\rangle_{0,2, d}^{\mathcal{X}} T_{j}\right) .
$$


1.3. Generating functions. Given an orbifold $\mathcal{X}$, Givental's (big) J-function is the first row vector of the fundamental solution matrix, obtained by pairing the solution vectors of the Dubrovin connection with 1.

$$
\begin{aligned}
J_{\text {big }}^{\mathcal{X}}(\mathbf{t}, z) & :=\sum_{i}\left(s_{i}(\mathbf{t}), 1\right)_{C R}^{\mathcal{X}} T^{i} \\
& =1+\sum_{d} \sum_{n \geq 0} \sum_{i} \frac{q^{d}}{n !}\left\langle\frac{T_{i}}{z-\psi_{1}}, 1, \mathbf{t}, \ldots, \mathbf{t}\right\rangle_{0,2+n, d}^{\mathcal{X}} T^{i} \\
& =1+\frac{\mathbf{t}}{z}+\sum_{d} \sum_{n \geq 0} \sum_{i} \frac{q^{d}}{n !}\left\langle\frac{T_{i}}{z\left(z-\psi_{1}\right)}, \mathbf{t}, \ldots, \mathbf{t}\right\rangle_{0,1+n, d}^{\mathcal{X}} T^{i},
\end{aligned}
$$

The last equality follows from the string equation. It is also easy to see that the fundamental solution matrix $S(\mathbf{t}, z)$ of (1.2.3) is equal to $z \nabla J_{\text {big. }}$. As such, $J_{b i g}$ encodes all information about quantum cohomology.

However, the big J-function is often impossible to calculate directly. In the non-orbifold Gromov-Witten theory, when the cohomology is generated by divisors, the small J-function proves much more computable, while powerful enough to solve many problems; see e.g. [13, 14]. The small Jfunction for a nonsingular variety $X$ is a function on $\mathbf{t} \in H^{2}(X)$ :

$$
\begin{aligned}
J_{\text {small }}^{X}(\mathbf{t}, z) & :=\left.J_{\text {big }}^{X}(\mathbf{t}, z)\right|_{\mathbf{t} \in H^{2}(X)} \\
& =e^{\mathbf{t} / z}\left(1+\sum_{d>0} \sum_{i} q^{d} e^{d \mathbf{t}}\left\langle\frac{T_{i}}{z-\psi_{1}}, 1\right\rangle_{0,2, d}^{X} T^{i}\right) .
\end{aligned}
$$

In orbifold theory, however, the Chen-Ruan cohomology is never generated by divisors except for trivial cases, due to the presence of the twisted sectors. Therefore, the knowledge of the small $J$-function alone is often not enough to reconstruct significant information about the orbifold quantum cohomology. (Note however that in Section 5 of [8] one way was found to circumvent this obstacle for weighted projective spaces.)

We propose the following definition of small J-matrix for orbifolds.

Definition 1.4. For $\mathbf{t} \in H^{2}(\mathcal{X})$, define $J_{g}^{\mathcal{X}}$ as the cohomology-valued function

$$
\begin{aligned}
\left.J_{g}^{\mathcal{X}}(\mathbf{t}, z)\right|_{\mathbf{t} \in H^{2}(\mathcal{X})} & :=\sum_{i}\left(\left.s_{i}(\mathbf{t})\right|_{\mathbf{t} \in H^{2}(X)}, \mathbb{1}_{g}\right)_{C R}^{\mathcal{X}} T^{i} \\
& =e^{\mathbf{t} / z}\left(\mathbb{1}_{g}+\sum_{d>0} \sum_{i} q^{d} e^{d \mathbf{t}}\left\langle\frac{T_{i}}{z-\psi_{1}}, \mathbb{1}_{g}\right\rangle_{0,2, d}^{\mathcal{X}} T^{i}\right),
\end{aligned}
$$

where $\mathbb{1}_{g}$ is the fundamental class on the component $\mathcal{X}_{g}$ of $I \mathcal{X}$.

The small J-matrix is the matrix-valued function

$$
J_{\text {small }}^{\mathcal{X}}(\mathbf{t}, z)=\left[J_{g, i}^{\mathcal{X}}(\mathbf{t}, z)\right]_{g \in G, i \in I}=\left[\left(J_{g}^{\mathcal{X}}(\mathbf{t}, z), T_{i}\right)_{C R}^{\mathcal{X}}\right]_{g \in G, i \in I},
$$

where $G$ is the index set of the components of $I \mathcal{X}, I$ the index for the basis $\left\{T_{i}\right\}_{i \in I}$ of $H_{C R}^{*}(\mathcal{X})$ and $J_{g, i}^{\mathcal{X}}(\mathbf{t}, z)$ the coefficient of $T^{i}$ in $J_{g}^{\mathcal{X}}(\mathbf{t}, z)$. 
Remark 1.5. We believe that the small $J$-matrix is the right replacement of the small $J$-function in the orbifold theory, for its computability and structural relevance.

Structurally equation (1.2.3) shows that one needs to specify "two-points" (i.e. a matrix) in the generating function in order to form the fundamental solutions of the Dubrovin connection. Ideally, one would like to get the full $|I| \times|I|$ fundamental solution matrix $S=z \nabla J_{\text {big }}$ restricted to $\mathbf{t} \in H^{2}(\mathcal{X})$. This would give all information about the small quantum cohomology. Unfortunately, a direct computation of $\left.S(\mathbf{t})\right|_{\mathbf{t} \in H^{2}(\mathcal{X})}$ is mostly out of reach in the orbifold theory.

In the (non-orbifold) case when $H^{*}(X)$ is generated by divisors, as shown by A. Givental, the small $J$-function is often enough to determine the essential information for small quantum cohomology. One can think of the small $J$-function as a submatrix of size $1 \times|I|$, indeed the first row vector, of $S$.

However, in the orbifold theory, the above matrix is not enough to determine useful information about small quantum cohomology except in the trivial cases. We believe that the smallest useful submatrix of $S$ is the small $J$-matrix (of size $|G| \times|I|$ ) defined above. We will show that it is both computable and relevant to the structure of orbifold quantum cohomology. In this paper we are able to calculate the small $J$-matrix of the toric orbifold $\mathcal{Y}=\left[\mathbb{P}^{4} / \bar{G}\right]$, and we use a sub-matrix of the small $J$-matrix $J_{\text {small }}^{\mathcal{W}}$ to fully describe the solution matrix $\left.S(\mathbf{t})\right|_{\mathbf{t} \in H^{2}(\mathcal{X})}$ of the mirror quintic $\mathcal{W}$.

\section{J-FUnCTION OF $\left[\mathbb{P}^{4} / \bar{G}\right]$}

2.1. Inertia orbifold of $\left[\mathbb{P}^{4} / \bar{G}\right]$. Let $\left[x_{0}, x_{1}, x_{2}, x_{3}, x_{4}\right]$ be the homogeneous coordinates of $\mathbb{P}^{4}$. Denote

$$
\zeta=\zeta_{5}:=e^{2 \pi \sqrt{-1} / 5}
$$

Let the group $\bar{G} \cong(\mathbb{Z} / 5 \mathbb{Z})^{3}$ be a (finite abelian) subgroup of the big torus of $\mathbb{P}^{4}$ acting via generators $e_{1}, e_{2}, e_{3}$ :

$$
\begin{aligned}
& e_{1}\left[x_{0}, x_{1}, x_{2}, x_{3}, x_{4}\right]=\left[\zeta x_{0}, x_{1}, x_{2}, x_{3}, \zeta^{-1} x_{4}\right] \\
& e_{2}\left[x_{0}, x_{1}, x_{2}, x_{3}, x_{4}\right]=\left[x_{0}, \zeta x_{1}, x_{2}, x_{3}, \zeta^{-1} x_{4}\right] \\
& e_{3}\left[x_{0}, x_{1}, x_{2}, x_{3}, x_{4}\right]=\left[x_{0}, x_{1}, \zeta x_{2}, x_{3}, \zeta^{-1} x_{4}\right] .
\end{aligned}
$$

Let $\mathcal{Y}=\left[\mathbb{P}^{4} / \bar{G}\right]$. As explained in the Introduction this orbifold plays an instrumental role in what follows so we give here a detailed presentation of its corresponding inertia orbifold.

The group $\bar{G}$ can be described alternatively as follows. Let

$$
G:=\left\{\left(\zeta^{r_{0}}, \ldots, \zeta^{r_{4}}\right) \mid \sum_{i=0}^{4} r_{i} \equiv 0(\bmod 5)\right\}
$$

and

$$
\bar{G} \cong G /\langle(\zeta, \ldots, \zeta)\rangle
$$


The $\bar{G}$-action on $\mathbb{P}^{4}$ comes from coordinate-wise multiplication. By a slight abuse of notation, we will represent a group element $g \in G$ by the power of $\zeta$ in each coordinate:

$$
G=\left\{\left(r_{0}, \ldots, r_{4}\right) \mid \sum_{i=0}^{4} r_{i} \equiv 0(\bmod 5), 0 \leq r_{i} \leq 4 \forall i\right\} .
$$

For an element $g \in G$, denote $[g]$ the corresponding element in $\bar{G}$.

Fix an element $\bar{g} \in \bar{G}$. Let $g=\left(r_{0}, \ldots, r_{4}\right) \in G$ be such that $[g]=\bar{g}$. Define

$$
I(g):=\left\{j \in\{0,1,2,3,4\} \mid r_{j}=0\right\},
$$

then

$$
\mathbb{P}_{g}^{4}:=\left\{x_{j}=0\right\}_{j \notin I(g)} \subset \mathbb{P}^{4}
$$

is a component of $\left(\mathbb{P}^{4}\right)^{\bar{g}}$. From this we see that each element $g \in G$ such that $[g]=\bar{g}$ corresponds to a connected component $\mathcal{Y}_{g}$ of $I \mathcal{Y}$ associated with $\mathbb{P}_{g}^{4} \subset\left(\mathbb{P}^{4}\right)^{\bar{g}}$. Note that if $g$ has no coordinates equal to zero then $\mathbb{P}_{g}^{4}$ is empty, and so is $\mathcal{Y}_{g}$. This gives us a convenient way of indexing components of $I \mathcal{Y}$.

We summarize the above discussions in the following lemma.

\section{Lemma 2.1.}

$$
I \mathcal{Y}=\coprod_{g \in S} \mathcal{Y}_{g}
$$

where

$$
\mathcal{Y}_{g}=\left\{(x,[g]) \in I \mathcal{Y} \mid x \in\left[\mathbb{P}_{g}^{4} / \bar{G}\right]\right\}
$$

is a connected component and $S$ denotes the set of all $g=\left(r_{0}, \ldots, r_{4}\right)$ such that at least one coordinate $r_{i}$ is equal to 0.

Consequently, a convenient basis $\left\{T_{i}\right\}$ for $H_{C R}^{*}(\mathcal{Y})$ is

$$
\bigcup_{g \in S}\left\{\mathbb{1}_{g}, \mathbb{1}_{g} \tilde{H}, \ldots, \mathbb{1}_{g} \tilde{H}^{\operatorname{dim}\left(\mathcal{Y}_{g}\right)}\right\} .
$$

2.2. J-functions. Recalling a basic fact about global quotient orbifolds, a map of orbifolds $f: \mathcal{C} \rightarrow\left[\mathbb{P}^{4} / \bar{G}\right]$ can be identified with a principal $\bar{G}$ bundle $C$, and a $\bar{G}$-equivariant map $\tilde{f}: C \rightarrow \mathbb{P}^{4}$ such that the following diagram commutes: 1

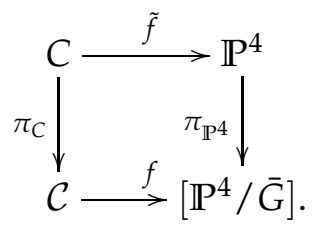

\footnotetext{
${ }^{1}$ Technically $\mathrm{f}$ is identified with an equivalence class of such objects.
} 
Lemma 2.2. (i) The map $\tilde{f}$ is representable if and only if $C$ is a nodal curve with each irreducible component a smooth variety.

(ii) There do not exist representable orbifold morphisms $f: \mathcal{C} \rightarrow \mathcal{Y}$ from a genus 0 orbifold curve $\mathcal{C}$ with only one orbifold marked point.

Proof. (i) follows immediately from the definition of representability.

(ii) follows from (i): If $\mathcal{C}$ is irreducible, this is because there do not exist smooth covers of genus 0 orbifold curves with only one point with nontrivial isotropy. An induction argument then shows that the same is true of reducible curves with only one orbifold marked point (we assume always that our nodes be balanced).

A line bundle on $\left[\mathbb{P}^{4} / \bar{G}\right]$ can be identified with a $\bar{G}$-equivariant line bundle on $\mathbb{P}^{4}$. Therefore, the Picard group on $\left[\mathbb{P}^{4} / \bar{G}\right]$ is a $\bar{G}$-extension of $\mathbb{Z}$. Let $L$ be any line bundle on $\left[\mathbb{P}^{4} / \bar{G}\right]$ such that $\pi_{\mathbb{P}^{4}}^{*} L=H$, where $H$ is the hyperplane class on $\mathbb{P}^{4}$. By (2.2.1), we have the following equality

$$
\int_{\mathcal{C}} f^{*}(L)=\frac{1}{125} \int_{C} \tilde{f}^{*}(H) \text {. }
$$

We define the degree of a map $f: \mathcal{C} \rightarrow \mathcal{Y}$ by

$$
d:=\frac{1}{125} \int_{C} \tilde{f}^{*}(H)
$$

This also allows us to determine necessary conditions on the triple $d$, $h=\left(r_{0}(h), \ldots, r_{4}(h)\right)$ and $g=\left(r_{0}(g), \ldots, r_{4}(g)\right)$ for

$$
\overline{\mathscr{M}}_{0, h, g}(\mathcal{Y}, d):=\overline{\mathscr{M}}_{0,2}(\mathcal{Y}, d) \cap e v_{1}^{-1}\left(\mathbb{1}_{h}\right) \cap e v_{2}^{-1}\left(\mathbb{1}_{g}\right)
$$

to be nonempty.

Proposition 2.3. The space $\overline{\mathscr{M}}_{0, h, g}(\mathcal{Y}, d)$ is nonempty only if

(i) $[h]=[g]^{-1}$ in $\bar{G}$;

(ii) $r_{i}(h)+r_{i}(g) \equiv 5 d(\bmod 5)$ or equivalently $\langle d\rangle=\left\langle\left(r_{i}(h)+r_{i}(g)\right) / 5\right\rangle$ for $0 \leq i \leq 4$.

Proof. We will first consider the case where the source curve is irreducible. Assume that there exists a map $\{f: \mathcal{C} \rightarrow \mathcal{Y}\}$ in $\overline{\mathscr{M}}_{0, h, g}(\mathcal{Y}, d)$ such that $\mathcal{C}$ is non-nodal. Consider the principal $\bar{G}$-bundle $\pi_{C}: C \rightarrow \mathcal{C}$. After choosing a generic base point $x \in \mathcal{C}$ and a point $\tilde{x}$ in $\pi_{C}^{-1}(x)$, we get a homomorphism $\phi: \pi_{1}(\mathcal{C}, x) \rightarrow \bar{G}$. We can specify generators $\rho_{1}$, and $\rho_{2}$ of $\pi_{1}(\mathcal{C}, x)$ such that $\rho_{i}$ is the class of loops wrapping once around $p_{i}$ in the counterclockwise direction. Then $\phi\left(\rho_{1}\right)=[h]$ and $\phi\left(\rho_{2}\right)=[g]$. Because $\rho_{1} \cdot \rho_{2}=1$ in $\pi_{1}(\mathcal{C}, x)$, it must be the case that $[h] \cdot[g]=1$ in $\bar{G}$. This proves (i) for $\mathcal{C}$ non-nodal.

Next we will show (ii) in the case where $\mathcal{C}$ is non-nodal. To see this, note that the only smooth connected cover of $\mathcal{C}$ is isomorphic to $\mathbb{P}^{1}$. This cover is degree $r:=|[h]|$, so $C$ must consist of $|\bar{G}| / r$ components, each isomorphic to $\mathbb{P}^{1}$. In the case $h=(0,0,0,0,0)$, this implies that $C$ has 125 components, and so $d$ is an integer. Thus Condition (ii) holds trivially. 
If $h \neq(0,0,0,0,0)$, then $r=5$. First note that (i) implies that $r_{i}(h)+$ $r_{i}(g)(\bmod 5)$ is the same for any $i$. Thus, we only need to prove the statement for one $i$. Let $\mu_{5}$ be the group generated by $[h]$. Let $C^{\prime} \cong \mathbb{P}^{1}$ be one component of $C$ and let

$$
f^{\prime}:=\left.\tilde{f}\right|_{C^{\prime}}: C^{\prime} \rightarrow \mathbb{P}^{4}
$$

be the $\mu_{5}$-equivariant morphism induced from the $\bar{G}$-equivariant morphism $\tilde{f}: C \rightarrow \mathbb{P}^{4} .\left(f^{\prime}\right)^{*}(\mathscr{O}(1))$ is a degree $5 d$ line bundle on $C^{\prime}=\mathbb{P}^{1}$. Therefore, any lifting of the torus action on $\mathbb{P}^{1}$ will have weights $(w, w+5 d)$ at the fibers of the 2 fixed points. Call these two fixed points $p_{1}^{\prime}$ and $p_{2}^{\prime}$. Since $\mu_{5}=\langle[h]\rangle$ is a subgroup of the torus, the characters of the $[h]$-action at the fibers of the 2 fixed points must be $\left(\zeta^{w}, \zeta^{w+5 d}\right)$, for some $w$ in $\{0, \ldots, 4\}$.

Let $q_{1}:=f^{\prime}\left(p_{1}^{\prime}\right)$ and $q_{2}:=f^{\prime}\left(p_{2}^{\prime}\right)$. By assumption, $q_{1} \in \mathbb{P}_{h^{\prime}}^{4} q_{2} \in$ $\mathbb{P}_{g}^{4}$. Choose an $i \in I(h)$ and $j \in I(g)$ such that $i \neq j, x_{i}\left(q_{1}\right) \neq 0$ and $x_{j}\left(q_{2}\right) \neq 0$. The action of $[h]$ on the fiber over $q_{1}$ and $q_{2}$ can be chosen to be $\left(\zeta^{r_{i}(h)}, \zeta^{-r_{j}(h)}\right)$. By the above weight/character arguments,

$$
r_{i}(h)-\left(-r_{j}(h)\right) \equiv 5 d(\bmod 5) .
$$

Since $j \in I(g)$ and $i \in I(h)$,

$$
r_{j}(h)=r_{j}(h)-r_{i}(h)=r_{i}(g)-r_{j}(g)=r_{i}(g),
$$

so we can rewrite the above as $r_{i}(h)+r_{i}(g) \equiv 5 d(\bmod 5)$.

The nodal case follows similarly. Consider a nodal curve $f: \mathcal{C} \rightarrow \mathcal{Y}$. Let $\mathcal{C}_{1}, \ldots, \mathcal{C}_{n}$ be the irreducible components connecting $p_{1}$ to $p_{2}$. It follows from Lemma 2.2, each of these components will have 2 orbifold points (at either nodes or marked points) and these will be the only points in $\mathcal{C}$ with nontrivial orbifold structure. The above calculation for irreducible components plus the condition that all nodes be balanced in this situation then implies the claim.

Once condition (i) is satisfied, the degree of maps allowed is thus determined by the quantity

$$
d(h, g):=\left\langle\left(r_{i}(h)+r_{i}(g)\right) / 5\right\rangle .
$$

Note that this number remains constant as $i$ varies.

We will define generating functions related to the $J$-functions $J_{g}^{\mathcal{Y}}$ which isolate the 2-point invariants of $\overline{\mathscr{M}}_{0, h, g}(\mathcal{Y}, d)$. Let

$$
S(d, h):=\left\{(b, k) \mid 0<b \leq d, \quad 0 \leq k \leq 4, \quad\langle b\rangle=r_{k}(h) / 5\right\},
$$

and let

$$
c(d, h):=|S(d, h)| .
$$

Given $h, g \in G$ such that $[h]=[g]^{-1}$, define

$$
Z_{h, g}:=\sum_{d} Q^{c(d, h)} \sum_{i}\left\langle\frac{T_{i}^{h}}{z-\psi_{1}}, \mathbb{1}_{g}\right\rangle_{0,2, d}^{\mathcal{Y}} T_{h}^{i},
$$


where $\left\{T_{i}^{h}\right\}$ is a basis for $H^{*}\left(\mathcal{Y}_{h}\right)$, and $\left\{T_{h}^{i}\right\}$ is the dual basis under the Chen-Ruan orbifold pairing. (The motivation behind this choice of exponent for $Q$ will become clear in what follows: it is chosen to simplify the recursion satisfied by our generating function). Notice that by the above lemma, the only degrees which contribute to $Z_{h, g}$ are $d$ such that $\langle d\rangle=$ $d(h, g)$. Finally, let

$$
Z_{g}:=\mathbb{1}_{g}+\sum_{\left\{h \mid[h]=[g]^{-1}\right\}} Z_{h, g} .
$$

Let $T=\left(\mathbb{C}^{*}\right)^{5}$ (or $\mathbb{C}^{*}$ ) act on $\mathbb{C}^{5}$ with (generic) weights $-\lambda_{0}, \ldots,-\lambda_{4}$. This induces an action on $\mathbb{P}^{4}$ and $\mathcal{Y}$. Furthermore there is an induced $T$ action on the inertia orbifold $I \mathcal{Y}$ and on $\overline{\mathscr{M}}_{0,2}(\mathcal{Y}, d)$. We will consider an equivariant analogue $Z_{g}^{T}$ of $Z_{g}$ defined by replacing the coefficients of $Z_{g}$ with their equivariant counterparts:

$$
Z_{h, g}^{T}:=\sum_{d, i} Q^{c(d, h)}\left\langle\frac{T_{i}^{h}}{z-\psi_{1}}, \mathbb{1}_{g}\right\rangle_{0,2, d}^{\mathcal{Y}, T} T_{h}^{i}, \quad Z_{g}^{T}:=\mathbb{1}_{g}+\sum_{\{h \mid[h]=[g]-1\}} Z_{h, g}^{T} .
$$

where $\left\{T_{i}^{h}\right\}$ is now a basis of the equivariant cohomology $H_{T}^{*}\left(\mathcal{Y}_{h}\right)$.

Consider the cohomology valued functions

$$
Y_{h, g}^{T}:=\sum_{\{d|d\rangle=d(h, g)\}} Q^{c(d, h)} \frac{\mathbb{1}_{h^{-1}}}{\prod_{(b, k) \in S(d, h)}\left(b z+H-\lambda_{k}\right)},
$$

where

$$
h^{-1}:=\left(-r_{0}(h), \ldots,-r_{4}(h)\right)(\bmod 5)
$$

As with $Z$, let

$$
Y_{g}^{T}:=\mathbb{1}_{g}+\sum_{\left\{h \mid[h]=[g]^{-1}\right\}} Y_{h, g}^{T} .
$$

Theorem 2.4. We have the equality in equivariant cohomology:

$$
Z_{g}^{T}=Y_{g}^{T}
$$

In particular, taking the nonequivariant limit, we conclude that $Z_{g}=Y_{g}$, (where $Y_{g}$ is the obvious non-equivariant limit of $Y_{g}^{T}$.)

Remark 2.5. For those who are familiar with the computation of the small $J$-function for toric manifolds [14], the generating functions $Z$, as indicated above, play the role of the $J$-function. The hypergeometric-type functions $Y$ then take the place of the I-function. Recall that one way of formulating the computation of genus zero GW invariants is to say that the J-function is equal to the $I$-function after a change of variables, called the mirror map. In the present case, the mirror map is trivial. 
2.3. Proof of Theorem 2.4. The proof follows from a localization argument similar in spirit to that in [14]. The strategy is to apply the Localization Theorem (after inverting the equivariant characters $\lambda_{0}, \ldots, \lambda_{4}$ in the ring $H_{C R, T}^{*}(\mathcal{Y})$ ) on the equivariant generating functions to determine a recursion satisfied by $Z_{g}^{T}$. This recursion relation in fact determines $Z_{g}^{T}$ up to the constant term in the Novikov variables. We then show that $Y_{g}^{T}$ satisfies the same recursion. Since $Z_{g}^{T}$ and $Y_{g}^{T}$ have the same initial term and the same recursion relation, $Z_{g}^{T}=Y_{g}^{T}$.

2.3.1. a lemma on $c(d, h)$. We will first explain the seemingly strange appearance of the exponents $c(d, h)$ in the definition of $Z_{h, g}$.

Lemma 2.6. Let

$$
m_{d}=\operatorname{dim}\left(\overline{\mathscr{M}}_{0, h, g}(\mathcal{Y}, d)\right),
$$

then if $[h]=[g]^{-1}$ and $\langle d\rangle=d(h, g)$, we have

$$
c(d, h)=m_{d}-\operatorname{dim}\left(\mathcal{Y}_{h}\right)+1 \text {. }
$$

Proof. The standard formula for virtual dimension gives

$$
m_{d}=5 d+3-\operatorname{age}(h)-\operatorname{age}(g) .
$$

Note that for any presentation $g=\left(r_{0}(g), \ldots, r_{4}(g)\right)$, age $(g)=\sum_{i=0}^{4} r_{i}(g) / 5$. Because $[h]=[g]^{-1}$, we have that

$$
r_{i}(g)-r_{j}(g) \equiv r_{j}(h)-r_{i}(h)(\bmod 5) .
$$

This allows us to write

$$
\frac{r_{k}(g)}{5}=\left\{\begin{array}{cc}
-r_{k}(h) / 5+d(h, g) & d(h, g) \geq r_{k}(h) / 5 \\
1-r_{k}(h) / 5+d(h, g) & d(h, g)<r_{k}(h) / 5
\end{array},\right.
$$

which gives

$$
\begin{aligned}
m_{d} & =5 d+3-5 d(h, g)-\left|\left\{k \mid d(h, g)<r_{k}(h) / 5\right\}\right| \\
& =5\lfloor d\rfloor+\left|\left\{k \mid d(h, g) \geq r_{k}(h) / 5\right\}\right|-2 .
\end{aligned}
$$

Now, for a fixed $k$,

$$
\left|\left\{b \mid 0 \leq b \leq d, \quad\langle b\rangle=r_{k}(h) / 5\right\}\right|=\left\{\begin{array}{cc}
\lfloor d\rfloor & d(h, g)<r_{k}(h) / 5 \\
1+\lfloor d\rfloor & d(h, g) \geq r_{k}(h) / 5
\end{array}\right\} .
$$

Summing over all $k$, we get that

$$
m_{d}=\left|\left\{(b, k) \mid 0 \leq b \leq d, \quad 0 \leq k \leq 4, \quad\langle b\rangle=r_{k}(h) / 5\right\}\right|-2 .
$$

Finally,

$$
\operatorname{dim}\left(\mathcal{Y}_{g}\right)=\left|\left\{k \mid 0=r_{k}(h) / 5\right\}\right|-1,
$$

which gives the desired equality. 
2.3.2. Setting up the localization. The action of $T$ on $\left.\overline{\mathscr{M}}_{0, h, g}(\mathcal{Y}, d)\right)$ allows us to reduce integrals on the moduli space to sums of integrals on the fixed point loci with respect to the torus action. As usual, this reduces us to considering integrals of certain graph sums (see [18]). The generating function $Z_{g}^{T}$ consists of integrals where the first insertion is the pull back of a class on

$$
\coprod_{\left\{h \mid[h]=[g]^{-1}\right\}} \mathcal{Y}_{h}
$$

We will now express $Z_{g}$ in terms of a new basis for this space which interacts nicely with the localization procedure. For each coordinate $0 \leq i \leq 4, i$ is in $I(h)$ for exactly one $h$ in $\left\{h \mid[h]=[g]^{-1}\right\}$. (Recall that the presentations $h \in\left\{h \mid[h]=[g]^{-1}\right\}$ index the fixed point sets of $\mathbb{P}^{4}$ with respect to $\left.[h]\right)$. Then for $i \in I(h)$, let $q_{i}$ be the $T$-fixed point of $\mathcal{Y}_{h}$ obtained by setting all coordinates $\{j \mid j \neq i\}$ equal to zero. Then, for $i \in I(h)$, let

$$
\phi_{i}=\mathbb{1}_{h} \cdot \prod_{j \in I(h)-i} H-\lambda_{j}
$$

If we pair $Z_{g}^{T}$ with $\phi_{i}$, we obtain the function

$$
Z_{i, g}^{T}=\frac{\delta^{i, I}(g)}{125}+\sum_{d} Q^{c(d, h)}\left\langle\frac{\phi_{i}}{z-\psi_{1}}, \mathbb{1}_{g}\right\rangle_{0,2, d}^{\mathcal{Y}, T}
$$

where $\delta^{i, I(g)}$ equals 1 if $i \in I(g)$ and 0 otherwise. The fixed point set of $\mathcal{Y}_{h}$ consists of $\left\{q_{j} \mid j \in I(h)\right\}$. Note that under the inclusion $i_{j}:\left\{q_{j}\right\} \rightarrow \mathcal{Y}_{h}, H$ pulls back to $\lambda_{j}$. Therefore $i_{j}^{*}\left(\phi_{i}\right)=0$ unless $i=j$. From this we see that the coefficients of $Z_{i, g}^{T}$ consist of integrals over graphs such that the first marked point is mapped to $q_{i}$.

We divide the remaining graphs into two types: those in which the first marked point is on a contracted component, and those in which the first marked point is on a noncontracted component.

Claim 2.7. There is no contribution from graphs of the first type.

Proof. The proof is a dimension count. We will show that the contributions from graphs of the first type must contain as a multiplicative factor integrals of the form $\int_{M} \Psi$ such that $\operatorname{deg}_{C}(\Psi)>\operatorname{dim}(M)$, and hence the vanishing claim.

The complex degree of $\phi_{i}$ is $\operatorname{dim}\left(\mathcal{Y}_{h}\right)$, so the invariant $\left\langle\phi_{i} \psi_{1}^{k}, \mathbb{1}_{g}\right\rangle_{0,2, d}^{\mathcal{Y}, T}$ vanishes unless $k \geq m_{d}-\operatorname{dim}\left(\mathcal{Y}_{h}\right)$. Thus we can simplify our expression for 
$Z_{i, g}^{T}:$

$$
\begin{aligned}
Z_{i, g}^{T} & =\frac{\delta^{i, I(g)}}{125}+\sum_{d} Q^{c(d, h)}\left\langle\frac{\phi_{i}}{z-\psi_{1}}, \mathbb{1}_{g}\right\rangle_{0,2, d}^{\mathcal{Y}, T} \\
& =\frac{\delta^{i, I(g)}}{125}+\sum_{d} Q^{c(d, h)} \frac{1}{z} \sum_{k=0}^{\infty}\left\langle\phi_{i}\left(\psi_{1} / z\right)^{k}, \mathbb{1}_{g}\right\rangle_{0,2, d}^{\mathcal{Y}, T} \\
& =\frac{\delta^{i, I(g)}}{125}+\sum_{d} Q^{c(d, h)} \frac{1}{z} \sum_{k=c(d, h)-1}^{\infty}\left\langle\phi_{i}\left(\psi_{1} / z\right)^{k}, \mathbb{1}_{g}\right\rangle_{0,2, d}^{\mathcal{Y}, T} \\
& =\frac{\delta^{i, I(g)}}{125}+\sum_{d}\left(\frac{Q}{z}\right)^{c(d, h)}\left\langle\frac{\phi_{i} \psi_{1}^{c(d, h)-1}}{1-\left(\psi_{1} / z\right)}, \mathbb{1}_{g}\right\rangle_{0,2, d}^{\mathcal{Y}, T} .
\end{aligned}
$$

Here the third equality follows from Lemma 2.6.

Now consider a fixed point graph $M_{\Gamma}$ such that $p_{1}$ is on a contracted component. At the level of virtual classes, we can write

$$
\left[M_{\Gamma}\right]=F(\Gamma) \cdot \prod_{k}\left[M_{v_{k}}\right]
$$

where each $M_{v_{k}}$ represents a contracted component of the graph isomorphic to a component of $\bar{M}_{0, n}\left(B \mathbb{Z}_{r}, 0\right)$, and $F(\Gamma)$ is a factor determined by $\Gamma$. Let $M_{v_{0}}$ be the component containing $p_{1} . M_{v_{0}}$ contains at most 2 orbifold marked points, and the number of non-orbifold marked points is restricted by $d$. In particular, each non-orbifold marked point corresponds to a (nonorbifold) edge of the dual graph. Each of these edges must have degree at least 1 , so if the total degree of the map is $d$, then there can be at most $\lfloor d\rfloor$ nontwisted marked points. Thus the dimension of $M_{v_{0}}$ is at most $\lfloor d\rfloor-1$. Now, the proof of Lemma 2.6 shows that

$$
c(d, h)-1=5\lfloor d\rfloor+\left|\left\{k \mid r_{k}(h) / 5 \leq d(h, g)\right\}\right|-2-\operatorname{dim}\left(\mathcal{Y}_{h}\right) .
$$

$\operatorname{But} \operatorname{dim}\left(\mathcal{Y}_{h}\right)$ is exactly $\left|\left\{k \mid r_{k}(h)=0\right\}\right|-1$, which implies that

$$
c(d, h)-1 \geq 5\lfloor d\rfloor-1 .
$$

If $d \geq 1$, the above quantity is strictly greater than $\lfloor d\rfloor-1$. Because there do not exist graphs such that $p_{1}$ is on a non-contracted component for $d<1$, we have that for $M_{\Gamma}, c(d, h)-1 \gtrless \operatorname{dim}\left(M_{v_{0}}\right)$. But $\psi_{1}^{c(d, I)-1}$ must therefore vanish on these graphs, proving the claim.

2.3.3. Contributions from a graph of the second type. Now let us consider the contribution to $\left\langle\frac{\phi_{i}}{z-\psi_{1}}, \mathbb{1}_{g}\right\rangle_{0,2, d}^{\mathcal{Y}, T}$ from a particular graph $\Gamma$ of the second type. In particular, we know that $p_{1}$ is on a noncontracted component. Call this component $\mathcal{C}_{0}$, and denote the rest of the graph $\Gamma^{\prime} . \Gamma^{\prime}$ and $\mathcal{C}_{0}$ connect at a node $p^{\prime}$, which maps to some $q_{k} \in \mathcal{Y}$. Let $d^{\prime}$ be the degree of one connected component of the principal $\bar{G}$-bundle above $\mathcal{C}_{0}$. We know from Proposition 2.3 that $\left\langle d^{\prime}\right\rangle=r_{k}(h) / 5$. By identifying $p^{\prime} \in \Gamma^{\prime}$ as a 
marked point (replacing $p_{1}$ on $\mathcal{C}_{0}$ ), we can view $M_{\Gamma^{\prime}}$ as a fixed point locus in $\overline{\mathscr{M}}_{0, h^{\prime}, g}\left(\mathcal{Y}, d-d^{\prime}\right)$, where $[h]=\left[h^{\prime}\right]$, but $r_{k}\left(h^{\prime}\right)=0$. Our plan will be to express integrals on $M_{\Gamma}$ in terms of integrals on $M_{\Gamma^{\prime}}$, thus reducing the calculation to one involving maps of strictly smaller degree. This will give us a recursion.

The factor $F(\Gamma)$ in Equation 2.3.1 is composed of three contributions: the automorphisms of the graph $\Gamma$ itself, a contribution from each edge of $\Gamma$ (the non-contracted components of curves in $M_{\Gamma}$ ), and a contribution from certain flags of $\Gamma$ (the nodes of curves in $M_{\Gamma}$ ). The edge corresponding to $\mathcal{C}_{0}$ maps to the line $q_{i k} \cong \mathbb{P}^{1} / \bar{G}$ connecting $q_{i}$ and $q_{k}$. (Note that the $\bar{G}$ action is a subgroup of the big torus $\left(\mathbb{C}^{*}\right)^{4}$ of $\mathbb{P}^{4}, \bar{G}$ naturally acts on $\left(\mathbb{C}^{*}\right)^{4}$ orbits.) The degree of the map upstairs is $5 d^{\prime}$. Thus there is a contribution of $1 /\left(5 d^{\prime}\right)$ to $F(\Gamma)$ from the automorphism of $M_{\Gamma}$ coming from rotating the underlying curve. The edge also contributes a factor of $1 / 25$ due to the fact that $q_{i k}$ is a $(\mathbb{Z} / 5 \mathbb{Z})^{2}$-gerbe. So the total contribution to $F(\Gamma)$ from the edge containing $p_{1}$ is $1 /\left(125 d^{\prime}\right)$. The contribution from the node $p^{\prime}$ is $125 / r$. (Recall $r=|[h]|$, which is equal to the order of the isotropy at $\left.p^{\prime}\right)$. There will be an additional factor of $r$ appearing when we examine deformations of $M_{\Gamma}$, thus canceling the $r$ in the denominator. We finally arrive at the relation

$$
\left[M_{\Gamma}\right]=F(\Gamma) \cdot \prod_{\text {vertices } v \in \Gamma}\left[M_{v}\right]=\frac{F\left(\Gamma^{\prime}\right)}{d^{\prime}} \cdot \prod_{\text {vertices } v \in \Gamma^{\prime}}\left[M_{v}\right]=\frac{1}{d^{\prime}}\left[M_{\Gamma^{\prime}}\right] .
$$

By examining the localization exact sequence (see [18]), we have the following identity:

$$
e\left(N_{\Gamma}\right)=\frac{e\left(H^{0}\left(\mathcal{C}_{0}, f^{*} T \mathcal{Y}\right)^{m}\right)\left(\text { node smoothing at } p^{\prime}\right)}{e\left(H^{0}\left(p^{\prime}, f^{*} T \mathcal{Y}\right)^{m}\right) e\left(H^{1}\left(\mathcal{C}_{0}, f^{*} T \mathcal{Y}\right)^{m}\right) e\left(\left(H^{0}\left(\mathcal{C}_{0}, T \mathcal{C}_{0}\right)^{m}\right)\right.} e\left(N_{\Gamma^{\prime}}\right)
$$

where $e$ denotes the equivariant Euler class, and as is standard we identify certain vector bundles with their fibers. Here the superscript $m$ denotes the moving part of the vector bundle with respect to the torus action. Let us calculate the factors in (2.3.2).

- (node smoothing at $p^{\prime}$ ): The node smoothing contributes a factor of

$$
\left(\frac{\lambda_{k}-\lambda_{i}}{r d^{\prime}}-\frac{\psi_{1}^{\prime}}{r}\right)=\frac{1}{r}\left(\frac{\lambda_{k}-\lambda_{i}}{d^{\prime}}-\psi_{1}^{\prime}\right),
$$

where $\psi_{1}^{\prime}$ is the $\psi$-class corresponding to $p_{1}^{\prime}$ on $M_{\Gamma}^{\prime}$. This factor of $r$ is what cancels with the previous factor mentioned above.

- $e\left(H^{0}\left(\mathcal{C}_{0}, T \mathcal{C}_{0}\right)^{m}\right)$ : Let $C$ be the principal $\bar{G}$-bundle over $\mathcal{C}_{0}$ induced from $\left.f\right|_{\mathcal{C}_{0}}: \mathcal{C}_{0} \rightarrow\left[\mathbb{P}^{4} / \bar{G}\right]$. As was argued in Proposition 2.3, $C$ consists of $(|\bar{G}| / r)$ copies of $\mathbb{P}^{1}$. Let $C_{0}$ be one of these copies. Then $C_{0}$ is a principal $\langle[h]\rangle$ bundle over $\mathcal{C}_{0}$ and

$$
H^{0}\left(\mathcal{C}_{0}, T \mathcal{C}_{0}\right)=H^{0}\left(C_{0}, T C_{0}\right)^{\langle[h]\rangle}
$$


The $\langle[h]\rangle$-invariant part of $H^{0}\left(C_{0}, T C_{0}\right)$ is one dimensional. It is fixed by the torus action, thus the moving part of $H^{0}\left(\mathcal{C}_{0}, T \mathcal{C}_{0}\right)$ is trivial and $e\left(H^{0}\left(\mathcal{C}_{0}, T \mathcal{C}_{0}\right)^{m}\right)=$ 1.

- $e\left(H^{1}\left(\mathcal{C}_{0}, f^{*} T \mathcal{Y}\right)^{m}\right)$ : Let $C_{0}$ be as in the previous bullet, then

$$
H^{1}\left(\mathcal{C}_{0}, f^{*} T \mathcal{Y}\right)=H^{1}\left(C_{0}, \tilde{f}^{*} T \mathbb{P}^{4}\right)^{\langle[h]\rangle}=0 .
$$

Therefore $e\left(H^{1}\left(\mathcal{C}_{0}, f^{*} T \mathcal{Y}\right)^{m}\right)=1$.

- $e\left(H^{0}\left(\mathcal{C}_{0}, f^{*} T \mathcal{Y}\right)^{m}\right)$ : To calculate this term, note that

$$
H^{0}\left(\mathcal{C}_{0}, f^{*} T \mathcal{Y}\right)^{m} \cong\left(H^{0}\left(C_{0}, \tilde{f}^{*} T \mathbb{P}^{4}\right)^{\langle[h]\rangle}\right)^{m} .
$$

We will look at the $\langle[h]\rangle$ invariant part of the short exact sequence

$$
0 \rightarrow \mathbb{C} \rightarrow H^{0}\left(\mathscr{O}_{C_{0}}\left(r d^{\prime}\right)\right) \otimes V \rightarrow H^{0}\left(\tilde{f}^{*} T \mathbb{P}^{4}\right) \rightarrow 0,
$$

where $\mathbb{P}^{4}=\mathbb{P}(V)$ and $V \cong \mathbb{C}^{5}$. The exact sequence comes from the pullback of the Euler sequence for $\mathbb{P}^{4}$ to $C_{0}$. (Note that the degree of $\tilde{f}: C_{0} \rightarrow$ $\mathbb{P}^{4}$ is $\left.r d^{\prime}\right)$. The action of $[h]$ on the first term in the sequence is trivial.

Recall that $\mathbb{P}(V)$ has coordinates $\left[x_{0}, \ldots, x_{4}\right]$. Let $[s, t]$ be homogeneous coordinates on $C_{0} \cong \mathbb{P}^{1}$, such that the preimage of $p_{1}$ in $C_{0}$ is $[0,1]$ and the preimage of $p^{\prime}$ in $C_{0}$ is $[1,0]$. Then the middle term of the sequence is spanned by elements of the form $s^{a} t^{b} \frac{\partial}{\partial x_{l}}$ where $0 \leq l \leq 4$ and $a+b=r d^{\prime}$. The action is given by

$$
[h] \cdot\left(s^{a} t^{b} \frac{\partial}{\partial x_{l}}\right)=e^{2 \pi \sqrt{-1}\left(-a+r_{l}(h)\right) / r_{s} s^{a} t^{b}} \frac{\partial}{\partial x_{l}},
$$

and so this summand is invariant under the $\langle[h]\rangle$-action if and only if $r_{l}(h) / r=$ $\langle a / r\rangle$. The $\mathbb{C}^{*}$-action on this term has weight

$$
\left(a / r d^{\prime}\right) \lambda_{k}+\left(b / r d^{\prime}\right) \lambda_{i}-\lambda_{l}
$$

so we finally arrive at

$$
\begin{aligned}
& e\left(H^{0}\left(\mathcal{C}_{0}, f^{*} T \mathcal{Y}\right)^{m}\right) \\
& =\prod_{\substack{\left\{(a, l) \mid 0 \leq a \leq r d^{\prime} \\
\backslash\left\{\leq l \leq 4 r_{r}(h) / r=\langle a / r\rangle\right\}\right.}}\left(\frac{a}{r d^{\prime}} \lambda_{k}+\frac{r d^{\prime}-a}{r d^{\prime}} \lambda_{i}-\lambda_{l}\right) \\
& =\prod_{\substack{\left\{(a, l) \mid 0 \leq a \leq r d^{\prime} \\
\backslash\left\{(0, i),\left(r d^{\prime}, k\right)\right\}\right.}}\left(a\left(\frac{\lambda_{k}-\lambda_{i}}{r d^{\prime}}\right)+\lambda_{i}-\lambda_{l}\right) .
\end{aligned}
$$

- $e\left(H^{0}\left(p^{\prime}, f^{*} T \mathcal{Y}\right)^{m}\right)$ : Similarly, the node $p^{\prime}$ is isomorphic to $B Z_{r}$, and each of the $|\bar{G}| / r$ points lying in the principal $\bar{G}$-bundle over $p^{\prime}$ is a principal $\langle[h]\rangle$-bundle over $p^{\prime}$. Thus $H^{0}\left(p^{\prime}, f^{*} T \mathcal{Y}\right)^{m} \cong\left(\left(T_{q_{k}} \mathbb{P}^{n}\right)^{\langle[h]\rangle}\right)^{m}$ and

$$
e\left(H^{0}\left(p^{\prime}, f^{*} T \mathcal{Y}\right)^{m}\right)=\prod_{l \in I\left(h^{\prime}\right) \backslash\{k\}}\left(\lambda_{k}-\lambda_{l}\right) .
$$


Finally note that $e v_{1}^{*}\left(\phi_{i}\right)=\prod_{l \in I(h)-i}\left(\lambda_{i}-\lambda_{l}\right)$. We can do one further simplification. On the graphs which we consider, namely those where $p_{1}$ is on a noncontracted component, $\psi_{1}$ restricts to $\frac{\lambda_{k}-\lambda_{i}}{d^{\prime}}$. (In fact $e\left(T_{p_{1}}^{*} \mathcal{C}\right) \cong$ $\frac{\lambda_{k}-\lambda_{i}}{r d^{\prime}}$, but because we are following the convention that $\psi$-classes are pulled back from the reification, we must multiply this by a factor of $r$ ).

These calculations plus (2.3.2) then give us the contribution to $\left\langle\frac{\phi_{i} \psi_{1}^{c(d, h)-1}}{1-\psi_{1} / z}, \mathbb{1}_{g}\right\rangle_{0,2, d}^{\mathcal{Y}, T}$ from the graph $M_{\Gamma}$ :

$$
\begin{aligned}
& \int_{\left[M_{\Gamma}\right]} \frac{e v_{1}^{*}\left(\phi_{i}\right) \psi_{1}^{c(d, I)-1}}{e\left(N_{\Gamma}\right)\left(1-\psi_{1} / z\right)}
\end{aligned}
$$

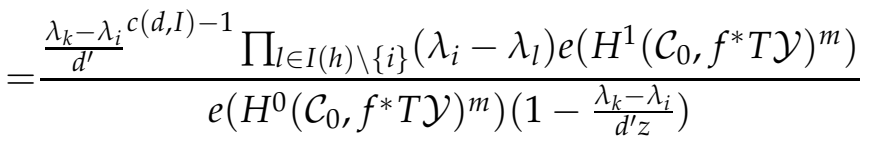

$$
\begin{aligned}
& \cdot \frac{1}{d^{\prime}} \int_{\left[M_{\Gamma}^{\prime}\right]} \frac{e\left(H^{0}\left(p^{\prime}, f^{*} T \mathcal{Y}\right)^{m}\right)}{\left(\text { node smoothing at } p^{\prime}\right) e\left(N_{\Gamma^{\prime}}\right)}
\end{aligned}
$$

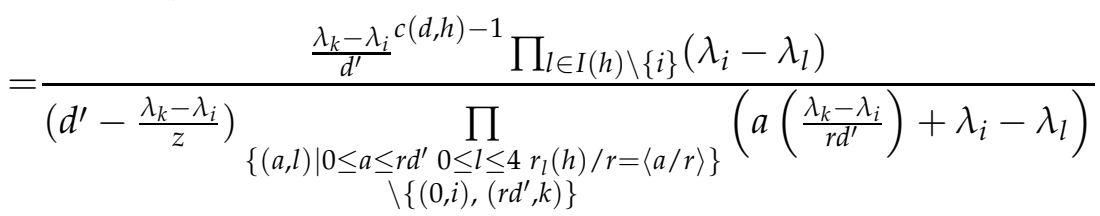

$$
\begin{aligned}
& \cdot \int_{\left[M_{\Gamma^{\prime}}\right]} \frac{\prod_{l \in I\left(h^{\prime}\right) \backslash\{k\}}\left(\lambda_{k}-\lambda_{l}\right)}{\left(\frac{\lambda_{k}-\lambda_{i}}{d^{\prime}}-\psi_{1}\right) e\left(N_{\Gamma^{\prime}}\right)} .
\end{aligned}
$$

2.3.4. Recursion relations. We will formulate the above computations into a recursion relation. To do that, the following regularity lemma is needed.

Lemma 2.8 (Regularity Lemma). $Z_{i, g}^{T}$ is an element of $\mathbb{Q}\left(\lambda_{i}, z\right)[[Q]]$. The coefficient of each $Q^{D}$ is a rational function of $\lambda_{i}$ and $z$ which is regular at $z=$ $\left(\lambda_{i}-\lambda_{j}\right) / k$ for all $j \neq i$ and $k \geq 1$.

Proof. This follows from a standard localization argument, see e.g. Lemma 11.2.8 in [9].

Using the Regularity Lemma, the above computation simplifies to

$$
\begin{aligned}
& \left(\left\langle\frac{\phi_{i} \psi_{1}^{c(d, h)-1}}{1-\psi_{1} / z}, \mathbb{1}_{g}\right\rangle_{0,2, d}^{\mathcal{Y}, T}\right)_{M_{\Gamma}} \\
& =\left.C_{d^{\prime}}^{i, k} \cdot\left(\frac{\lambda_{k}-\lambda_{i}}{d^{\prime}}\right)^{c(d, h)-1-\left(c\left(d^{\prime}, h\right)-1\right)} \cdot\left(\left\langle\frac{\phi_{k}}{z-\psi_{1}}, \mathbb{1}_{g}\right\rangle_{0,2, d-d^{\prime}}^{\mathcal{Y}, T}\right)_{M_{\Gamma^{\prime}}}\right|_{z \mapsto \frac{\lambda_{k}-\lambda_{i}}{d^{\prime}}},
\end{aligned}
$$

where

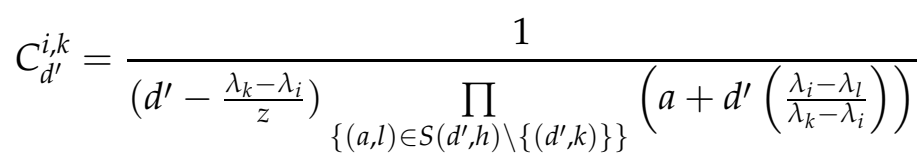


and $(-)_{M_{\Gamma}}$ means the contribution of the fixed component $M_{\Gamma}$ to the expression in parentheses.

Due to the fact that $r_{k}(h) / 5=\left\langle d^{\prime}\right\rangle$, one can check that

$$
c(d, h)-c\left(d^{\prime}, h\right)=c\left(d-d^{\prime}, h^{\prime}\right)
$$

(see (2.3.4)). We arrive at the expression

$$
\left.C_{d^{\prime}}^{i, k} \cdot\left(Q^{c\left(d-d^{\prime}, k\right)}\left\langle\frac{\phi_{k}}{z-\psi_{1}}, \mathbb{1}_{g}\right\rangle_{0,2, d-d^{\prime}}^{\mathcal{Y}, T}\right)_{M_{\Gamma^{\prime}}}\right|_{z \mapsto \frac{\lambda_{k}-\lambda_{i}}{d^{\prime}}, Q \mapsto \frac{\lambda_{k}-\lambda_{i}}{d^{\prime}}} \cdot
$$

After summing over all possible graphs, we obtain the recursion:

$$
Z_{i, g}^{T}=\frac{\delta^{i, I(g)}}{125}+\left.\sum_{\left\{\left(d^{\prime}, k\right) \mid \frac{r_{k}(h)}{5}=\left\langle d^{\prime}\right\rangle, k \neq i, d^{\prime} \neq 0\right\}}\left(\frac{Q}{z}\right)^{c\left(d^{\prime}, h\right)} C_{d^{\prime}}^{i, k} \cdot Z_{k, g}^{T}\right|_{z \mapsto \frac{\lambda_{k}-\lambda_{i}}{d^{\prime}}, Q \mapsto \frac{Q}{z} \frac{\lambda_{k}-\lambda_{i}}{d^{\prime}}} \cdot
$$

Although we have suppressed this in the notation, recall that in the above summand, $h$ is the presentation such that $\phi_{i} \in \mathcal{Y}_{h}(i \in I(h))$.

We will now turn our attention to $Y_{g}^{T}$. Let us define the function $Y_{i, g}^{T}$ analogously to that of $Z_{i, g}^{T}$,

$$
Y_{i, g}^{T}:=\left(\phi_{i}, Y_{g}^{T}\right)_{C R}^{\mathcal{Y}}
$$

For $i \in I(h)$,

$$
Y_{i, g}^{T}=\frac{1}{125}\left(\delta^{i, I(g)}+\sum_{\langle d\rangle=d(h, g)} Q^{c(d, h)} \frac{1}{\prod_{(b, k) \in S(d, h)}\left(b z+\lambda_{i}-\lambda_{k}\right)}\right) .
$$

Claim 2.9. $Y_{i, g}^{T}$ satisfy the same recursion as $Z_{i, g}^{T}$ in (2.3.3). 
Proof. Consider the summand of $Y_{i, g}^{T}$ of degree $c(d, h)$ in $Q$, which we will denote $\left(Y_{i, g}^{T}\right)^{c(d, h)}$.

$$
\begin{aligned}
& \left(Y_{i, g}^{T}\right)^{c(d, h)}=\frac{1}{125}\left(\frac{Q}{z}\right)^{c(d, h)} \frac{1}{\prod_{(b, k) \in S(d, h)}\left(b+\left(\lambda_{i}-\lambda_{k}\right) / z\right)} \\
& =\frac{1}{125}\left(\frac{Q}{z}\right)^{c(d, h)} \sum_{\left\{(b, k) \mid r_{k}(h) / 5=\langle b\rangle, k \neq i, b \neq 0\right\}} \frac{1}{\left(b+\left(\lambda_{i}-\lambda_{k}\right) / z\right)} \\
& \cdot \frac{1}{\prod_{(m, l) \in S(d, h) \backslash\{(b, k)\}}\left(b\left(\lambda_{i}-\lambda_{l}\right) /\left(\lambda_{k}-\lambda_{i}\right)+m\right)} \\
& =\frac{1}{125}\left(\frac{Q}{z}\right)^{c(d, h)} \sum_{\left\{(b, k) \mid r_{k}(h) / 5=\langle b\rangle, k \neq i, b \neq 0\right\}} \\
& \left(\frac{1 /\left(b+\left(\lambda_{i}-\lambda_{k}\right) / z\right)}{\prod_{\{(m, l) \in S(d, h) \backslash\{(b, k)\} \mid m \leq b\}}\left(b\left(\lambda_{i}-\lambda_{l}\right) /\left(\lambda_{k}-\lambda_{i}\right)+m\right)}\right. \\
& \left.\cdot \frac{1}{\prod_{\{(m, l) \in S(d, h) \backslash\{(b, k)\} \mid m>b\}}\left(b\left(\lambda_{i}-\lambda_{l}\right) /\left(\lambda_{k}-\lambda_{i}\right)+m\right)}\right) .
\end{aligned}
$$

The last product from above can be rewritten as

$$
\prod_{(n, l) \in S\left(d-b, h^{\prime}\right)}\left(n+b \frac{\lambda_{k}-\lambda_{l}}{\lambda_{k}-\lambda_{i}}\right),
$$

where $h^{\prime}$ is chosen such that $[h]=\left[h^{\prime}\right]$ and $k \in I\left(h^{\prime}\right)$. To see this note that if $(b, k)$ and $(m, l)$ are both in $S(d, h)$, then by definition $r_{k}(h) / 5=\langle b\rangle$ and $r_{l}(h) / 5=\langle m\rangle$. If $k \in I\left(h^{\prime}\right)$, then

$$
\begin{aligned}
& \frac{r_{l}\left(h^{\prime}\right)}{5}=\frac{r_{l}\left(h^{\prime}\right)}{5}-\frac{r_{k}\left(h^{\prime}\right)}{5} \\
\equiv & \frac{r_{l}(h)}{5}-\frac{r_{k}(h)}{5} \equiv\langle m\rangle-\langle b\rangle \equiv\langle m-b\rangle(\bmod 1) .
\end{aligned}
$$

In other words $r_{l}\left(h^{\prime}\right) / 5=\langle m-b\rangle$. This proves that if $(b, k) \in S(d, h)$, and $h^{\prime}$ is chosen as above, then for pairs $(m, l)$ with $b<m \leq d$,

$$
(m, l) \in S(d, h) \text { if and only if }(m-b, l) \in S\left(d-b, h^{\prime}\right) .
$$

We arrive at the relation

$$
\begin{aligned}
& \left(Y_{i, g}^{T}\right)^{c(d, h)} \\
= & \left.\sum_{\left\{(b, k) \mid r_{k}(h) / 5=\langle b\rangle, k \neq i, b \neq 0\right\}}\left(\frac{Q}{z}\right)^{c(b, h)} C_{b}^{i, k}\left(Y_{k, g}^{T}\right)^{c\left(d-b, h^{\prime}\right)}\right|_{z \mapsto \frac{\lambda_{k}-\lambda_{i}}{b}, Q \mapsto \frac{Q}{z} \frac{\lambda_{k}-\lambda_{i}}{b}} .
\end{aligned}
$$


We conclude that $Y_{i, g}^{T}$ satisfy the same recursion as $Z_{i, g}^{T}$.

The recursion relation and initial conditions imply $Y_{i, g}^{T}=Z_{i, g}^{T}$. The proof of Theorem 2.4 is now complete.

Remark 2.10. As a corollary one may easily obtain an explicit formula for the small $J$-matrix $J_{\text {small }}^{\mathcal{Y}}(t, z)$ by isolating coefficients of the various $Z_{g}^{\mathcal{Y}}$. We give an explicit expression for certain specified rows of $J_{\text {small }}^{\mathcal{Y}}(t, z)$ in Corollary 3.8 .

\section{A MODEL OF THE MIRROR QUINTIC $\mathcal{W}$}

3.1. Fermat quintic and its mirror. Let $M \subset \mathbb{P}^{4}$ be the Fermat quintic defined by the equation $Q_{0}(x)=x_{0}^{5}+x_{1}^{5}+x_{3}^{5}+x_{4}^{5}+x_{5}^{5}$

$$
M:=\left\{Q_{0}(x)=0\right\} \subset \mathbb{P}^{4} \text {. }
$$

The Greene-Plesser's mirror construction [19] gives the mirror orbifold as the quotient stack

$$
\mathcal{W}:=[M / \bar{G}] .
$$

Note that the $\bar{G}$-action on $\mathbb{P}^{4}(2.1 .1)$ preserves the quintic equation $Q_{0}(x)$ and therefore induces an action on $M$. Equivalently,

$$
\mathcal{W}=\left\{Q_{0}=0\right\} \subset \mathcal{Y}=\left[\mathbb{P}^{4} / \bar{G}\right] \text {. }
$$

Remark 3.1. Since in this section we will only be interested in the GromovWitten theory ( $A$ model), which is deformation invariant, we will only speak of the mirror orbifold instead of the mirror family.

Recall in Lemma 2.1 the inertia orbifold of $\mathcal{Y}=\left[\mathbb{P}^{4} / \bar{G}\right]$ is indexed by $g \in G$. For a particular $g$, the dimension of $\mathcal{Y}_{g}$ is equal to $\left|\left\{j \mid r_{j}=0\right\}\right|-1$, and can be identified with a linear subspace of $\mathcal{Y}$. The age shift of $\mathcal{Y}_{g}$ is age $(g)=\sum_{i=0}^{4} r_{i} / 5$.

The inertia orbifold of the mirror quintic $\mathcal{W}$ can be described by that of $\mathcal{Y}$. $\mathcal{W}$ intersects nontrivially with $\mathcal{Y}_{g}$ exactly when $\left|\left\{j \mid r_{j}=0\right\}\right| \geq 2$. (that is, $\operatorname{dim} \mathcal{Y}_{g} \geq 1$.) Let

$$
\bar{S}:=\left\{g=\left(r_{0}, \ldots, r_{4}\right) \in G|2 \leq|\left\{j \mid r_{j}=0\right\} \mid\right\} .
$$

(Note that $\bar{S}$ contains $e=(0, \ldots, 0)$.) Then

$$
I \mathcal{W}=\coprod_{g \in \bar{S}} \mathcal{W}_{g}, \quad \mathcal{W}_{g}:=\mathcal{W} \cap \mathcal{Y}_{g}
$$

All nontrivial intersections are transverse, so

$$
\operatorname{dim}\left(\mathcal{W}_{g}\right)=\operatorname{dim}\left(\mathcal{Y}_{g}\right)-1=\left|\left\{j \mid r_{j}=0\right\}\right|-2
$$

It follows that the age shift of $\mathcal{W}_{g}$ is equal to the age shift of $\mathcal{Y}_{g}$. The cohomology of $\mathcal{W}$ is given by

$$
H_{C R}^{*}(\mathcal{W})=\bigoplus_{g \in \bar{S}} H^{*-2 \operatorname{age}(g)}\left(\mathcal{W}_{g}\right)
$$


In the sequel, we will only be interested in the subring of $H_{C R}^{*}(\mathcal{W})$ consisting of classes of even (real) degree. We will denote this ring as $H_{C R}^{\text {even }}(\mathcal{W})$. It can be checked via a direct calculation that if $i: \mathcal{W} \hookrightarrow \mathcal{Y}$ is the inclusion,

$$
H_{C R}^{\text {even }}(\mathcal{W})=i^{*} H_{C R}^{*}(\mathcal{Y}) \text {. }
$$

Conventions 3.2. Let $H$ be the hyperplane class on $\mathbb{P}^{4}$. By an abuse of notation, we will denote $H$ any fixed choice of $L$ on $\mathcal{Y}$ such that $\pi_{\mathbb{P}^{4}}^{*}(L)=H$, where $\pi_{\mathbb{P}^{4}}$ was defined in (2.2.1). We will also denote $H$ the induced class on $\mathcal{W}$. Even though there are as many as $|\bar{G}|$ choices of $L$, they are topologically equivalent and will serve the same purpose in our discussion.

A convenient basis $\left\{T_{i}\right\}$ for $H_{C R}^{\text {even }}(\mathcal{W})$ is

$$
\bigcup_{g \in \bar{S}}\left\{\mathbb{1}_{g}, \mathbb{1}_{g} H, \ldots, \mathbb{1}_{g} H^{\operatorname{dim}\left(\mathcal{W}_{g}\right)}\right\} .
$$

We also note that $H_{C R}^{\text {even }}(\mathcal{W}) \subset H_{C R}^{*}(\mathcal{W})$ is a self-dual subring with respect to the Poincaré pairing of $H_{C R}^{*}(\mathcal{W})$. Furthermore, this basis is selfdual (up to a constant factor). Given $g=\left(r_{0}, \ldots, r_{4}\right) \in S$, let

$$
g^{-1}:=\left(-r_{1}, \ldots,-r_{4}\right)(\bmod 5) \text {. }
$$

Then the Poincaré dual elements can be easily calculated:

$$
\left(\mathbb{1}_{g} H^{k}\right)^{\vee}=25\left(\mathbb{1}_{g^{-1}} H^{\operatorname{dim}\left(\mathcal{W}_{g}\right)-k}\right)
$$

\subsection{J-functions of $\mathcal{W}$.}

Conventions 3.3. By the matrix $J$-function of $\mathcal{W}$, we will mean the matrix consisting of the collection of $H_{C R}^{\text {even }}(\mathcal{W})$-valued functions with variable $\mathbf{t}=$ $t H$.

$$
J_{g}^{\mathcal{W}}(t, z):=e^{t H / z}\left(\mathbb{1}_{g}+\sum_{d, i} q^{d} e^{d t}\left\langle\frac{T_{i}}{z-\psi_{1}}, \mathbb{1}_{g}\right\rangle_{0,2, d}^{\mathcal{W}} T^{i}\right),
$$

where the basis $\left\{T_{i}\right\}$ is for $H_{C R}^{\text {even }}(\mathcal{W})$, as in (3.1.2). Here as in Section 2 , by the degree $d$ of a map $f: \mathcal{C} \rightarrow \mathcal{W}$ we mean

$$
d:=\int_{\mathcal{C}} f^{*}(H)
$$

Note that if we extend the basis $\left\{T_{i}\right\}$ to full basis of $H_{C R}^{*}(\mathcal{C})$, the classes of odd (real) degree will not contribute to $J_{g}^{\mathcal{W}}(t, z)$, and thus (3.2.1) is equal to the $J_{g}$-function of (1.3.1).

As has been shown in Proposition 2.3, for an orbi-curve $\mathcal{C}$ with two marked points, the degree must be a multiple of $1 / 5$. Recall also from Proposition 2.3 that the only nonzero contribution to the terms in $J_{g}^{\mathcal{W}}$ comes from elements $T_{i}$ supported on some $\mathcal{W}_{h}$ such that $[h]=\left[g^{-1}\right]$. From the definition of $\bar{S}$, it is required that

$$
\left|\left\{j \mid r_{j}=0\right\}\right| \geq 2, \quad \sum r_{j} \equiv 0(\bmod 5) .
$$


We will enumerate all possible cases.

It follows from the conditions (3.2.2) that $\left|\left\{j \mid r_{j}=0\right\}\right|$ must be equal to 2, 3 or 5 . That is, $\operatorname{dim}\left(\mathcal{W}_{g}\right)$ is equal to 0,1 or 3 .

If $\operatorname{dim}\left(\mathcal{W}_{g}\right)=3, g=e=(0,0,0,0,0)$ and $\mathbb{1}_{e}=1$. The only basis elements which contribute to $J_{e}^{\mathcal{W}}$ come from the nontwisted sector. We have

$$
J_{e}^{\mathcal{W}}(t, z)=e^{t H / z}\left(1+\sum_{d>0} q^{d} e^{d t}\left\langle\frac{H^{i}}{z-\psi_{1}}, 1\right\rangle_{0,2, d}^{\mathcal{W}}\left(25 H^{3-i}\right)\right)
$$

If $\operatorname{dim}\left(\mathcal{W}_{g}\right)=1$, then up to a permutation of the entries, $g=\left(0,0,0, r_{1}, r_{2}\right)$ with $r_{1} \neq r_{2}$. By definition of $\bar{S}$, other than $g$ there is no $h \in \bar{S}$ such that $[h]=[g]$. Therefore, the two basis elements which contribute nontrivially to $J_{g}^{\mathcal{W}}$ are $\mathbb{1}_{g^{-1}}$ and $\mathbb{1}_{g^{-1}} H$. We arrive at

$$
\begin{aligned}
& J_{g}^{\mathcal{W}}(t, z)=e^{t H / z}\left(\mathbb{1}_{g}+\right. \\
& \left.\sum_{d>0} q^{d} e^{d t}\left(\left\langle\frac{\mathbb{1}_{g-1}}{z-\psi_{1}}, \mathbb{1}_{g}\right\rangle_{0,2, d}^{\mathcal{W}}\left(25 \mathbb{1}_{g} H\right)+\left\langle\frac{\mathbb{1}_{g^{-1}} H}{z-\psi_{1}}, \mathbb{1}_{g}\right\rangle_{0,2, d}^{\mathcal{W}}\left(25 \mathbb{1}_{g}\right)\right)\right) .
\end{aligned}
$$

If $\operatorname{dim}\left(\mathcal{W}_{g}\right)=0$, then up to a permutation of the entries, $g=\left(0,0, r_{1}, r_{1}, r_{2}\right)$, with $r_{1} \neq r_{2}$. There is only one other $g_{1} \in \bar{S}$ such that $\left[g_{1}\right]=[g]$, namely, $g_{1}=\left(-r_{1},-r_{1}, 0,0, r_{2}-r_{1}\right)(\bmod 5)$. The two basis elements which contribute nontrivially to the invariants of $J_{g}^{\mathcal{W}}$ are $\mathbb{1}_{g^{-1}}$ and $\mathbb{1}_{\left(g_{1}\right)^{-1}}$. Thus we can express $J_{g}^{\mathcal{W}}(t, z)$ as

$$
\begin{aligned}
& J_{g}^{\mathcal{W}}(t, z)=e^{t H / z}\left(\mathbb{1}_{g}+\right. \\
& \left.\sum_{d>0} q^{d} e^{d t}\left(\left\langle\frac{\mathbb{1}_{g^{-1}}}{z-\psi_{1}}, \mathbb{1}_{g}\right\rangle_{0,2, d}^{\mathcal{W}}\left(25 \mathbb{1}_{g}\right)+\left\langle\frac{\mathbb{1}_{\left(g_{1}\right)-1}}{z-\psi_{1}}, \mathbb{1}_{g}\right\rangle_{0,2, d}^{\mathcal{W}}\left(25 \mathbb{1}_{g_{1}}\right)\right)\right) .
\end{aligned}
$$

Thus for each twisted component $\mathcal{W}_{g}$, the $J$-function $J_{g}^{\mathcal{W}}$ has two components.

We will relate the functions $J_{g}^{\mathcal{W}}$ to certain hypergeometric functions, called I-functions. To start with, let us introduce "bundled-twisted" GromovWitten invariants. Let $E \rightarrow \mathcal{X}$ be a line bundle over the orbifold $\mathcal{X}$. We 
have the following diagram

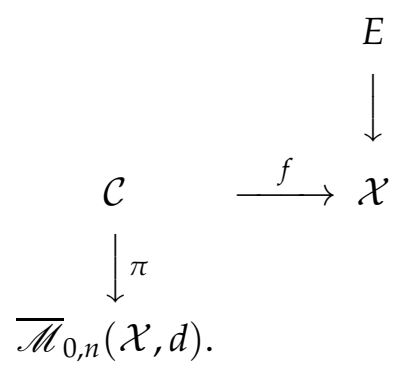

The E-twisted Gromov-Witten invariants are defined to be

$$
\left\langle\alpha_{1} \psi^{k_{1}}, \ldots, \alpha_{n} \psi^{k_{n}}\right\rangle_{0, n, d}^{\mathcal{X}, \mathrm{tw}}=\int_{\left.\left[\mathscr{M}_{0, n}(\mathcal{X}, d)\right]\right]^{v i r}} \prod_{i=1}^{n} e v_{i}^{*}\left(\alpha_{i}\right) \psi_{i}^{k_{i}} \cup e\left(E_{0, n, d}\right),
$$

where

$$
E_{o, n, d}:=\pi_{*} f^{*}(E)
$$

and $e\left(E_{0, n, d}\right)$ is the Euler class of the $K$-class. We can define a twisted pairing on $H_{C R}^{*}(\mathcal{X} ; \Lambda)$ by

$$
\left(\alpha_{1}, \alpha_{2}\right)_{C R}^{\mathcal{X}, \mathrm{tw}}=\int_{\mathcal{X}} \alpha_{1} \cup I^{*}\left(\alpha_{2}\right) \cup e(E) .
$$

With this, we can define a twisted $J$-function

$$
J^{\mathcal{X}, \mathrm{tw}}(\mathbf{t}, z)=1+\mathbf{t} / z+\sum_{d} \sum_{n \geq 0} \sum_{i} \frac{q^{d}}{n !}\left\langle\frac{T_{i}}{z-\psi_{1}}, 1, \mathbf{t}, \ldots, \mathbf{t}\right\rangle_{0,2+k, d}^{\mathcal{X}, \mathrm{tw}} T^{i} .
$$

Here $T_{i}$ is a basis for $H_{C R}^{*}(\mathcal{X} ; \Lambda)$ and $T^{i}$ is the dual basis with respect to the twisted pairing.

The twisted invariants are related to invariants on the hypersurface. In our case, $\mathcal{X}=\mathcal{Y}=\left[\mathbb{P}^{4} / \bar{G}\right]$, and $E=\mathscr{O}(5) \rightarrow \mathcal{Y}$. It is easy to see that $E_{0, n, d}=R^{0} \pi_{*} f^{*}(\mathscr{O}(5))$ is a vector bundle. The embedding $i: \mathcal{W} \hookrightarrow \mathcal{Y}$ induces a morphism $\iota: \overline{\mathscr{M}}_{0, n}(\mathcal{W}, d) \hookrightarrow \overline{\mathscr{M}}_{0, n}(\mathcal{Y}, d)$. It is well-known that

$$
\iota_{*}\left[\overline{\mathscr{M}}_{0, n}(\mathcal{W}, d)\right]^{\mathrm{vir}}=e\left(E_{0, n, d}\right) \cap\left[\overline{\mathscr{M}}_{0, n}(\mathcal{Y}, d)\right]^{\mathrm{vir}} .
$$

A proof can be found in e.g. [10]. (That proof, given in the nonorbifold setting there, can be readily modified to the orbifold setting.) This relates the twisted invariants on $\mathcal{Y}$ to the invariants on $\mathcal{W}$. Assume that $\mathbf{t}$ is restricted to $H_{C R}^{\text {even }}(\mathcal{Y})$, then

$$
J^{\mathcal{W}}(\mathbf{t}, z)=i^{*} J^{\mathcal{Y}, t w}(\mathbf{t}, z)
$$

Let us now further restrict $\mathbf{t}$ to $H_{C R}^{2}(\mathcal{Y})$. In our setting we may write an element of $H_{C R}^{2}(\mathcal{Y})$ as

$$
\mathbf{t}=t H+\sum_{\{g \mid \operatorname{age}(g)=1\}} t^{g} \mathbb{1}_{g}
$$


Write the $J$-function of $\mathcal{Y}$ as

$$
J^{\mathcal{Y}}(\mathbf{t})=\sum_{d} q^{d} J_{d}^{\mathcal{Y}}(\mathbf{t})
$$

For each $d$, define the modification factor

$$
M_{d}^{E / \mathcal{W}}:=\prod_{m=1}^{5 d}(5 H+m z)
$$

(Note that we have taken the $\lambda=0$ limit in [7].)

Definition 3.4. Define the twisted I-function by

$$
I^{E}(\mathbf{t}):=\sum_{d} q^{d} M_{d}^{E / \mathcal{W}} J_{d}^{\mathcal{Y}}(\mathbf{t})
$$

Write

$$
\begin{aligned}
I^{E}(\mathbf{t}, z)= & I_{e}^{E}(t, z)+\frac{1}{z}\left(\sum_{\{g \mid \operatorname{age}(g)=1\}} t^{g} I_{g}^{E}(t, z)\right) \\
& +\frac{1}{z}\left(\sum_{\left\{g_{1}, g_{2} \mid \operatorname{age}\left(g_{i}\right)=1\right\}} t^{g_{1}} t^{g_{2}} I_{g_{1}, g_{2}}^{E}(t, z)+\ldots\right) .
\end{aligned}
$$

For $g$ such that age $(g) \leq 1$ (including $g=e$ ), define the $A$ model hypergeometric functions

$$
I_{g}^{A}(t, z)=i^{*}\left(I_{g}^{E}(t, z)\right) .
$$

Theorem 3.5. Given $g=\left(r_{0}, \ldots, r_{4}\right)$ such that the age shift of $\mathcal{W}_{g}$ is at most 1 , there exist functions $F_{0}(t), G_{0}(t)$, and $H_{g}(t)$, determined explicitly by $I_{g}^{E}(t, z)$ such that $F_{0}$ and $H_{g}(g \neq 0)$ are invertible, and

$$
J_{g}^{\mathcal{W}}(\tau(t), z)=\frac{I_{g}^{A}(t, z)}{H_{g}(t)} \quad \text { where } \tau(t)=\frac{G_{0}(t)}{F_{0}(t)} .
$$

Remark 3.6. In the statement of the theorem, $F_{0}(t)$ and $G_{0}(t)$ do not depend on $g$, so the mirror map $t \mapsto \tau(t)=G_{0}(t) / F_{0}(t)$ is well defined.

3.3. Proof of Theorem 3.5. There are two key ingredients in the proof. The first one is the version of quantum Lefschetz hyperplane theorem (QLHT) for orbifolds proved in [7]. By Equation (3.1.1), $\mathcal{W}$ is a hyperplane section of $\mathcal{Y}$ and hence $J^{\mathcal{W}}$ can be calculated by QLHT. Corollary 5.1 in [7] in particular implies the following:

Theorem 3.7 ([7]). Let the setting be as above, with $E=\mathscr{O}(5) \rightarrow \mathcal{Y}$. Then

$$
I^{E}(\mathbf{t}, z)=F(\mathbf{t})+\frac{G(\mathbf{t})}{z}+O\left(z^{-2}\right)
$$


for some $F$ and $G$ with $F$ scalar valued and invertible, and

$$
J^{\mathcal{Y}, \mathrm{tw}}(\tau(\mathbf{t}), z)=\frac{I^{E}(\mathbf{t}, z)}{F(\mathbf{t})} \quad \text { where } \tau(\mathbf{t})=\frac{G(\mathbf{t})}{F(\mathbf{t})}
$$

The second ingredient is the explicit formula of $J_{g}^{\mathcal{Y}}$ from Section 2. Note that we are only concerned with those $g$ such that $i^{*} \mathbb{1}_{g} \neq 0$ and age $\left(\mathbb{1}_{g}\right) \leq 1$. Therefore only those $J_{g}^{\mathcal{Y}}$ are listed. The following is a straightforward corollary of Theorem $2.4,(2.2 .2)$ and $(2.2 .3)$ by equating the terms $Q^{c(d, h)} \mathbb{1}_{h^{-1}} H^{k}$ of $Z_{g}$ with the terms $q^{d} e^{d t} \mathbb{1}_{h^{-1}} H^{k}$ of $J_{g}^{\mathcal{Y}}$.

Corollary 3.8. The functions $J_{g}^{\mathcal{Y}}(t, z)$ are given by the following formulas.

(i) If $g=e=(0,0,0,0,0)$,

$$
J_{e}^{\mathcal{Y}}=e^{t H / z}\left(1+\sum_{\langle d\rangle=0} q^{d} e^{d t} \frac{1}{\prod_{\substack{0<b \leq d \\\langle b\rangle=0}}(b z-H)^{5}}\right) .
$$

(ii) If $g=\left(0,0,0, r_{1}, r_{2}\right)$, let $g_{1}=\left(-r_{1},-r_{1},-r_{1}, 0, r_{2}-r_{1}\right)(\bmod 5)$ and let $g_{2}=\left(-r_{2},-r_{2},-r_{2}, r_{1}-r_{2}, 0\right)(\bmod 5)$. Then

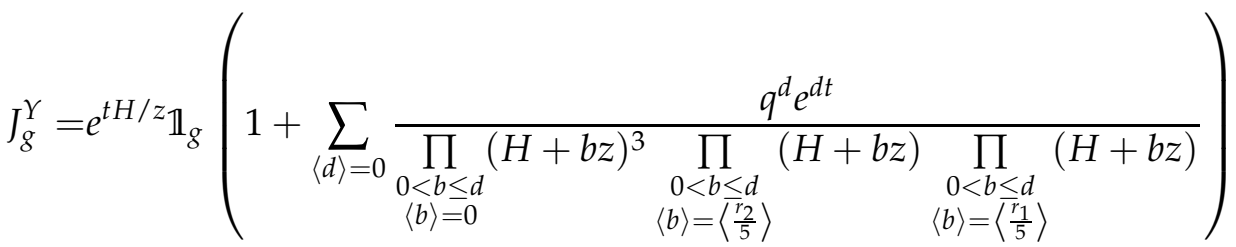

$$
\begin{aligned}
& +e^{t H / z} \mathbb{1}_{g_{1}}\left(\sum_{\langle d\rangle=\left\langle\frac{r_{1}}{5}\right\rangle} \frac{q^{d} e^{d t}}{\prod_{\substack{0<b \leq d \\
\langle b\rangle=\left\langle\frac{1}{5}\right\rangle}}(H+b z)^{3} \prod_{\substack{0<b \leq d \\
\langle b\rangle=0}}(H+b z) \prod_{\substack{0<b \leq d \\
\langle b\rangle=\left\langle\frac{2 r_{1}}{5}\right\rangle}}(H+b z)}\right)
\end{aligned}
$$

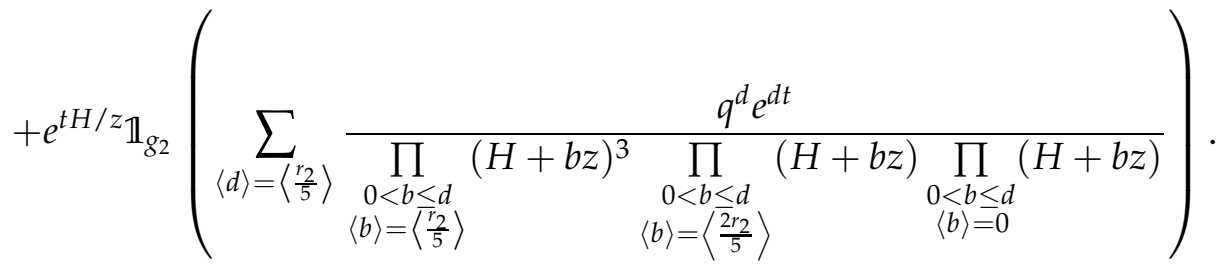


(iii) If $g=\left(0,0, r_{1}, r_{1}, r_{2}\right)$, let $g_{1}=\left(-r_{1},-r_{1}, 0,0, r_{2}-r_{1}\right)(\bmod 5)$ and let $g_{2}=\left(-r_{2},-r_{2}, r_{1}-r_{2}, r_{1}-r_{2}, 0\right)(\bmod 5)$. Then

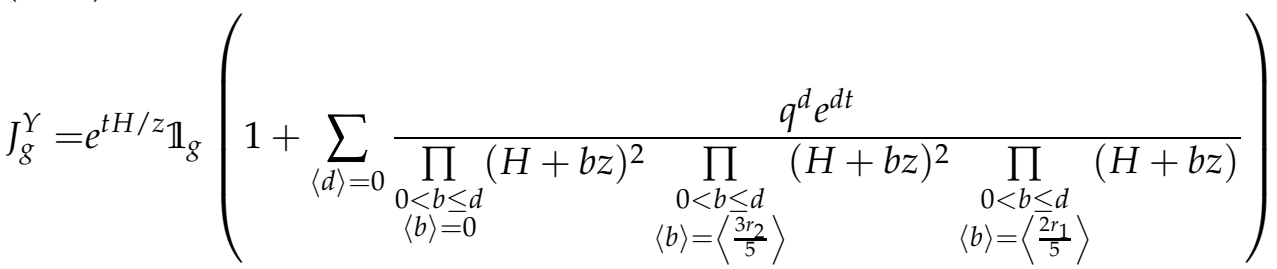

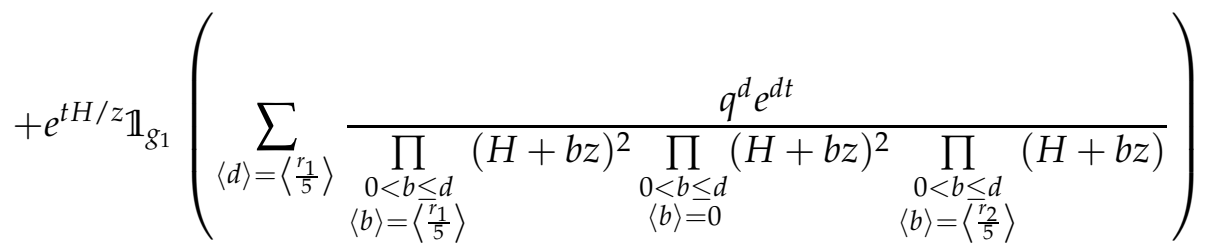

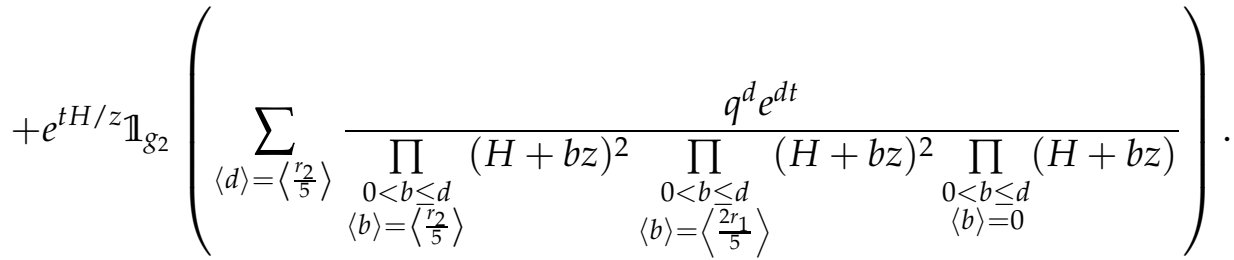

In fact, due to the age requirement, there are only two choices in case (ii) up to permutation: $\left(r_{1}, r_{2}\right)=(2,3)$ or $(1,4)$. In case (iii), only $\left(r_{1}, r_{2}\right)=(1,3)$ or $(2,1)$ are possible.

Lemma 3.9. There are scalar valued functions $F_{0}(t), G_{0}(t)$ and $G_{g}(t)$ for each $g$ with age $(g)=1$, such that

$$
i^{*}\left(I^{E}(\mathbf{t}, z)\right)=F_{0}(t)+\frac{G_{0}(t) H}{z}+\sum_{\operatorname{age}(g)=1} \frac{t^{g} G_{g}(t) \mathbb{1}_{g}}{z}+R,
$$

where $R$ denotes the remainder, consisting of terms with either the degrees in $t^{g}$ 's greater or equal to 2 or the degree in $z^{-1}$ greater or equal to 2 . In other words, if we write $G(\mathbf{t})$ from (3.3.1) as

$$
G(\mathbf{t})=\overline{G_{0}}(\mathbf{t}) H+\sum_{g} \overline{G_{g}}(\mathbf{t}) \mathbb{1}_{g}
$$

and denote $\mathrm{O}(2)$ the terms with the degrees in $t^{g}$ 's greater or equal to 2, then

$$
F(\mathbf{t})=F_{0}(t)+O(2), \quad \overline{G_{0}}(\mathbf{t})=G_{0}(t)+O(2), \quad \overline{G_{g}}(t)=t^{g} G_{g}(t)+O(2) .
$$

Proof. The proof of this lemma follows from Corollary 3.8 together with the following observations. First, in case (ii) $i^{*}\left(\mathbb{1}_{g_{1}}\right)=i^{*}\left(\mathbb{1}_{g_{2}}\right)=0$ due to dimensional reasons. Similarly with $i^{*}\left(\mathbb{1}_{g_{2}}\right)=0$ in case (iii). Secondly, in case (iii) the $\mathbb{1}_{g_{1}}$ term has higher $z^{-1}$ power: The modification factor contributes terms of $z^{5 d}$ plus lower order (in $z$ ) terms. $i^{*} J_{g}^{\mathcal{Y}}$ contributes $z^{-(5 d+1)}$ 
plus higher order (in $z^{-1}$ ) terms. The combined contribution goes to the remainder $R$.

With all this preparation, it is easy to prove Theorem 3.5

Proof of Theorem 3.5. Start by pulling back the equation (3.3.2) to $\mathcal{W}$. Setting all $t^{g}=0$ we get (3.2.10) for the case $g=e$ if we let $H_{e}=F_{0}$ :

$$
I_{e}^{A}(t)=i^{*} I_{e}^{E}(t)=\left.i^{*} I^{E}(\mathbf{t})\right|_{\mathbf{t}=t H} .
$$

Here by $\mathbf{t}=t H$ we mean that setting all $t^{g}=0$ in (3.2.7). In the case $g \neq e$, take the partial derivative of (3.3.2) with respect to $t^{g}$ and then set all $t^{g}=0$. Note that from (3.2.8), we have

$$
I_{g}^{A}(t)=i^{*} I_{g}^{E}(t)=\left.z \frac{\partial}{\partial t^{g}} i^{*} I^{E}(\mathbf{t})\right|_{\mathbf{t}=t H}
$$

By Lemma 3.9 all the "extra terms" vanish and (3.2.10) follows for $g \neq e$ after letting $H_{g}(t)=G_{g}(t)$. The proof is now complete.

\section{Periods And PiCARD-Fuchs EQuATIONS}

The theory of variation of Hodge structures (VHS) is closely related to the $B$ model of a Calabi-Yau variety $X$, which encodes information about the deformations of complex structures on $X$. By the local Torelli theorem for Calabi-Yau's, the Kodaira-Spencer spaces inject to the tangent spaces of period domains and one can investigate the deformations of $X$ via VHS, which can be described by a system of flat connections on cohomology vector bundles.

For the benefit of the readers who come from the GWT side of mirror symmetry, we give a brief and self-contained summary of the parts of VHS theory which are related to our work: the Gauss-Manin connection and the associated notions of the period matrix and Picard-Fuchs equations. For a more detailed introduction the reader may consult [16], [15].

4.1. Gauss-Manin connections, periods, and Picard-Fuchs equations. Over a smooth family of projective varieties $\pi: \mathscr{X} \rightarrow S$ of relative dimension $n$, we can consider the higher direct image sheaf (tensored with $\mathscr{O}_{S}$ ) on $S$ :

$$
R^{n} \pi_{*} \mathbb{C} \otimes \mathscr{O}_{S} .
$$

The fiber over a point $t \in S$ of this sheaf is $H^{n}\left(X_{t}\right)$. This sheaf is locally free, and is naturally endowed with a flat connection $\nabla^{G M}$, the Gauss-Manin connection. It can be defined in terms of the flat sections given by the lattice $R^{n} \pi_{*} \mathbb{Z}$ in $R^{n} \pi_{*} \mathbb{C} \rightarrow S$, a local system. The Hodge filtration can be described fiberwise by

$$
\left(\mathscr{F}^{p}\right)_{t} \cong \oplus_{a \geq p} H^{a, n-a}\left(X_{t}\right) .
$$

We will be particularly interested in the case when the base $S$ is one dimensional. Suppose now $S$ is an open curve and the family $\pi$ extends to a flat family over a proper curve $\bar{S}$. The vector bundle $R^{n} \pi_{*} \mathbb{C} \otimes \mathscr{O}_{S}$ extends 
to a vector bundle $\mathscr{H} \rightarrow \bar{S}$ whose fiber over $t$ in $S$ consists of the middle cohomology group $H^{n}\left(X_{t}\right)$. While it is not true that $\nabla^{G M}$ extends to a connection on all of $\mathscr{H}$, the singularities which arise are at worst a regular singularities [11]. This means that after choosing local coordinates, the connection matrix acquires at worst a logarithmic pole at $t=0$. Nevertheless we may still speak of flat (multi-valued) sections of $\nabla^{G M}$, controlled by the monodromy.

Let $\left\{\gamma_{i}\right\}$ be a basis of $H_{n}\left(X_{t_{0}}\right)$. Since $\pi: \mathscr{X} \rightarrow S$ is smooth, it is a locally trivial fibration and $n$-cycles $\gamma_{i}$ can be extended to locally constant cycles $\gamma_{i}(t)$. Let $\omega_{t}$ be a (local) section of $\mathscr{H}$. The functions $\int_{\gamma(t)} \omega_{t}$ are called the periods and by the local constancy of $\gamma(t)$

$$
\frac{d}{d t}\left(\int_{\gamma(t)} \omega_{t}\right)=\int_{\gamma(t)} \nabla_{t}^{G M_{S}}(t)
$$

The periods satisfy the Picard-Fuchs equations, defined as follows. Taking successive derivatives of $\omega_{t}$ with respect to the connection gives a sequence of sections

$$
\omega_{t}, \nabla_{t}^{G M} \omega_{t}, \ldots,\left(\nabla_{t}^{G M}\right)^{k} \omega_{t}, \ldots
$$

Because the rank of $\mathscr{H}$ is finite, for some $k$ there will exist a relation between these sections of the form

$$
\left(\nabla_{t}^{G M}\right)^{k} \omega_{t}+\sum_{i=0}^{k-1} f_{i}(t)\left(\nabla_{t}^{G W}\right)^{i} \omega_{t}=0
$$

The corresponding differential equation

$$
\left(\left(\frac{d}{d t}\right)^{k}+\sum_{i=0}^{k-1} f_{i}(t)\left(\frac{d}{d t}\right)^{i}\right)\left(\int_{\gamma(t)} \omega_{t}\right)=0
$$

is the Picard-Fuchs equation for $\omega_{t}$. The situation when the dimension of $S$ is greater than one is essentially the same, but (4.1.1) is replaced by a PDE.

Let $\left\{\phi_{i}\right\}_{i \in I}$ be a basis of sections of $\mathscr{H}$. Then if $\left\{\gamma_{i}\right\}_{i \in I}$ is a basis of locally constant $n$-cycles, we can write the fundamental solution matrix of the Gauss-Manin connection in coordinates as

$$
S=\left(s_{i j}\right) \text { with } s_{i j}=\int_{\gamma_{j}} \phi_{i} .
$$

With this choice of basis, we see that the $i^{\text {th }}$ row of $S$ gives the periods for the section $\phi_{i}$.

Remark 4.1. In the literature, often (but not always) the term periods are reserved for the case when $\phi(t)$ is a (holomorphic) $n$-form, i.e. a section of $\mathscr{F}^{n}$, and Picard-Fuchs equations only for periods in this restricted sense. Here, we choose to use these terms in a more general sense defined above. Note, however, by the results in [2], for Calabi-Yau threefolds the general Picard-Fuchs equations can be determined from the restricted ones. 
Remark 4.2. Let $U$ denote the Kuranishi space of the Calabi-Yau $n$-fold $X$. For the purpose of this paper, we use the term (genus zero part of) B model of $X$ to denote the vector bundle $\mathscr{H} \rightarrow U$ with the natural (flat) fiberwise pairing and the Gauss-Manin connection.

4.2. Griffiths-Dwork method. Let us assume now that the family $X_{t}$ is a family of hypersurfaces defined by homogeneous polynomials $Q_{t}$ of degree $d$ in $\mathbb{P}^{n+1}$. In this case the Griffiths-Dwork method can be employed to explicitly calculate the Picard-Fuchs equations. We summarize the relevant results of [15] here.

The method relies on Griffiths' work in [15] showing that one can calculate the period integrals on $X_{t}$ as one of rational forms on $\mathbb{P}^{n+1}$. For the time being, let us fix $t$ and suppress it in the notation. Griffiths first shows that in fact any class $\Omega$ in $H^{n+1}\left(\mathbb{P}^{n+1} \backslash X\right)$ can be represented in cohomology by a rational $n+1$ form. In particular, let $\Omega_{0}$ be the canonical $n+1$-form on $\mathbb{P}^{n+1}: \Omega_{0}=\sum_{i=0}^{n+1}(-1)^{i} x_{i} d x_{0} \cdots d \hat{x}_{i} \cdots d x_{n+1}$. We can represent $\Omega$ by a rational form with poles in $X$,

$$
\Omega=\frac{P(x)}{Q(x)^{k}} \Omega_{0}
$$

where $P(x)$ is a homogeneous polynomial with degree $k d-(n+2)$.

The rational $n+1$ forms are then related to regular $n$ forms on $X$ via the residue map. More precisely, let $A_{k}^{n}(X)$ denote the space of rational $(n+1)$-forms on $\mathbb{P}^{n+1}$ with poles of order at most $k$ on $X$, and let

$$
\mathcal{H}_{k}(X):=A_{k}^{n+1}(X) / d A_{k-1}^{n}(X) .
$$

This gives an obvious filtration

$$
\mathcal{H}_{1}(X) \subset \mathcal{H}_{2}(X) \subset \cdots \subset \mathcal{H}_{n+1}(X)=: \mathcal{H}(X) .
$$

This description of rational forms interacts nicely with the Hodge filtration $F^{p}$ of the primitive classes. Griffiths proves that the following diagram

$$
\begin{array}{ccccccc}
\mathcal{H}_{1}(X) & \subset & \mathcal{H}_{2}(X) & \subset & \cdots & \subset & \mathcal{H}_{n+1}(X) \\
\downarrow \operatorname{Res} & & \downarrow \operatorname{Res} & & & & \downarrow \text { Res } \\
F^{n} & \subset & F^{n-1} & \subset & \cdots & \subset & F^{0}
\end{array}
$$

is commutative, and that each vertical arrow is surjective. In particular, $\mathcal{H}_{k+1}(X) / \mathcal{H}_{k}(X) \cong F^{n-k} / F^{n-k+1}$.

Now, for each $n$-cycle $\gamma$ in $H_{n}(X)$, let

$$
T: H_{n}(X) \rightarrow H_{n+1}\left(\mathbb{P}^{n+1} \backslash X\right)
$$

be the tube map such that $T(\gamma)$ is a sufficiently small $S^{1}$-bundle around $\gamma$ in $\mathbb{P}^{n+1} \backslash X$. Griffiths then shows that the tube map is surjective in general and also injective when $n$ is odd.

Theorem 4.3. All primitive classes on $X$ can be represented as residues of rational forms on $\mathbb{P}^{n+1}$ with poles on $X$. This representation is unique when $n$ is odd. 
This follows from the surjectivity/injectivity of Res and $T$, as well as the residue formula

$$
\frac{1}{2 \pi i} \int_{T(\gamma)} \Omega=\int_{\gamma} \operatorname{Res}(\Omega)
$$

Next Griffiths relates the rational forms to the Jacobian ring. Let $J(Q)=$ $\left\langle\partial Q / \partial x_{0}, \ldots, \partial Q / \partial x_{n+1}\right\rangle$ be the Jacobian ideal of $Q$.

Theorem 4.4.

$$
\mathbb{C}\left[x_{0}, \ldots, x_{n+1}\right]_{d k-n-1} / J(Q) \cong F^{n-k} / F^{n+1-k} \cong P H^{n-k, k}(V) .
$$

The key relationship between rational forms is given by the following formula ((4.5) in [15])

$$
\frac{\Omega_{0}}{Q(x)^{k}} \sum_{j=0}^{n+1} B_{j}(x) \frac{\partial Q(x)}{\partial x_{j}}=\frac{1}{k-1} \frac{\Omega_{0}}{Q(x)^{k-1}} \sum_{j=0}^{n+1} \frac{\partial B_{i}(x)}{\partial x_{j}}+d \phi,
$$

where $\phi \in A_{k-1}^{n}$. Thus, the order of the pole of a form $\frac{P(x)}{Q(x)^{k}} \Omega_{0}$ can be lowered if and only if $P(x)$ is contained in $J(Q)$. Thus by identifying the form $\operatorname{Res}\left(\frac{P(x)}{Q(x)^{k}} \Omega_{0}\right)$ with the homogeneous polynomial $P$, one obtains the isomorphism.

The above results allow one to explicitly calculate the Picard-Fuchs equations for certain families of forms $\omega_{t}$ on $X_{t}$. As before, $X_{t}$ is a family of hypersurfaces defined by degree $d$ homogeneous polynomials $Q_{t}$. Then we can represent a family of forms as $\omega_{t}=\operatorname{Res}\left(\frac{P_{t}(x)}{Q_{t}(x)^{k}} \Omega_{0}\right)$. Let $\gamma_{t}$ be a locally constant $n$ cycle as before, then

$$
\begin{aligned}
\frac{\partial}{\partial t} \int_{\gamma_{t}} \omega_{t} & =\frac{\partial}{\partial t} \int_{\gamma_{t}} \operatorname{Res}\left(\frac{P_{t}(x)}{Q_{t}(x)^{k}} \Omega_{0}\right)=\frac{\partial}{\partial t} \int_{T\left(\gamma_{t}\right)} \frac{P_{t}(x)}{Q_{t}(x)^{k}} \Omega_{0} \\
& =\int_{T\left(\gamma_{t}\right)} \frac{\partial}{\partial t}\left(\frac{P_{t}(x)}{Q_{t}(x)^{k}} \Omega_{0}\right)=\int_{\gamma_{t}} \operatorname{Res}\left(\frac{\partial}{\partial t}\left(\frac{P_{t}(x)}{Q_{t}(x)^{k}} \Omega_{0}\right)\right) .
\end{aligned}
$$

The third equality follows because a small change in $T(\gamma(t))$ will not change its homology class. In other words, letting $\nabla^{G M}$ denote the Gauss-Manin connection,

$$
\nabla_{t}^{G M} \operatorname{Res}\left(\frac{P_{t}(x)}{Q_{t}(x)^{k}} \Omega_{0}\right)=\operatorname{Res}\left(\frac{\partial}{\partial t}\left(\frac{P_{t}(x)}{Q_{t}(x)^{k}} \Omega_{0}\right)\right),
$$

allowing one to obtain the Picard-Fuchs equations of $\omega_{t}$ via explicit calculations of the polynomials (in the Jacobian rings). An explicit example is given in the next section.

\section{B MODEL OF THE FERMAT QUINTIC $M$}

We now turn to the specific case of the Fermat quintic threefold $M$ in $\mathbb{P}^{4}$. It has been shown that the Hodge diamonds of $M$ and $\mathcal{W}$ are mirror symmetric

$$
h^{p, q}(M)=h^{3-p, q}(\mathcal{W})
$$


In particular, the deformation family of $\mathcal{W}$ is one-dimensional while for $M$ the deformation is 101 dimensional.

Recall in our study of the $A$ model of $\mathcal{W}$, we restrict the Dubrovin connection (i.e. Frobenius structure) to to the "small" parameter $t$ corresponding to the hyperplane class $H$. In the following discussions of the complex moduli of $M$, we will also study the full period matrix for the Gauss-Manin connection, but restricted to a particular deformation parameter.

Let

$$
Q_{\psi}(x)=x_{0}^{5}+x_{1}^{5}+x_{2}^{5}+x_{3}^{5}+x_{4}^{5}-\psi x_{0} x_{1} x_{2} x_{3} x_{4}
$$

and define the family $M_{\psi}=\left\{Q_{\psi}(x)=0\right\} \subset \mathbb{P}^{4}$. When writing the PicardFuchs equations it will later become convenient to the coordinate change $t=-5 \log (\psi)$.

5.1. Picard-Fuchs equations for $M_{\psi}$. In the specific case of the family $M_{\psi}$, there is a "diagrammatic technique", pioneered in [4] and refined in [12], which utilizes the symmetry of $Q_{\psi}$ and $P$ to simplify the bookkeeping.

The starting point is the equation (4.2.3). Consider the rational form

$$
\omega_{\psi}=\frac{P(x)}{Q_{\psi}(x)^{k}} \Omega_{0}, \quad P(x)=x_{0}^{r_{0}} \cdots x_{4}^{r_{4}}, \quad \text { with } \sum_{i=0}^{4} r_{i}=5(k-1) .
$$

Fix $i$ between 0 and 4 , and set $B_{j}=\delta_{i j} x_{i} P(x)$ for $0 \leq j \leq 4$. Noting that

$$
\frac{\partial}{\partial x_{i}} Q_{\psi}(x)=5 x_{i}^{4}-\psi x_{0} \cdots \hat{x}_{j} \cdots x_{4}
$$

and applying (4.2.3) with these choices of $B_{j}$ (and $k$ replaced by $k+1$ ), we arrive at

$$
5 \int_{T(\gamma)} \frac{\left(x_{i}^{5}\right) P}{Q_{\psi}^{k+1}} \Omega_{0}-\psi \int_{T(\gamma)} \frac{\left(x_{0} \ldots x_{4}\right) P}{Q_{\psi}^{k+1}} \Omega_{0}=\frac{1+r_{i}}{k} \int_{T(\gamma)} \frac{P}{Q_{\psi}^{k}} \Omega_{0}
$$

for any choice of cycle $\gamma \in H_{n}(X)$. Note, however, that there is a degenerate case in the above setting: in the case when $P(x)$ is independent of $x_{i}$, let $B_{j}=\delta_{i j} P(x)$. Then in (4.2.3) we get

$$
5 \int_{T(\gamma)} \frac{\left(x_{i}^{4}\right) P}{Q_{\psi}^{k+1}} \Omega_{0}-\psi \int_{T(\gamma)} \frac{\left(x_{0} \ldots \hat{x}_{i} \ldots x_{4}\right) P}{Q_{\psi}^{k+1}} \Omega_{0}=0 .
$$

We can interpret this equation as allowing $r_{i}=-1$ in (5.1.1).

Furthermore, $\frac{\partial}{\partial \psi} Q_{\psi}=-x_{0} \cdots x_{4}$, and so we have the relationship

$$
\frac{\partial}{\partial \psi} \int_{T(\gamma)} \frac{P}{Q_{\psi}^{k}} \Omega_{0}=k \int_{T(\gamma)} \frac{\left(x_{0} \cdots x_{4}\right) P}{Q_{\psi}^{k+1}} \Omega_{0} .
$$

The authors in [4, 12] apply (5.1.1) (5.1.2) and (5.1.3) recursively to get relations of the periods, hence the Picard-Fuchs equations. For convenience of bookkeeping, one can keep track of the polynomial $P(x)$ by its exponents 
$\left(r_{0}, \ldots, r_{4}\right)$. (5.1.1) can be understood symbolically as a relation between $\left(r_{0}, \ldots, r_{4}\right),\left(r_{0}, \ldots, r_{i}+5, \ldots, r_{4}\right)$ and $\left(r_{0}+1, \ldots, r_{4}+1\right)$.

Consider for example the case $P=1$ corresponding to $(0, \ldots, 0)$. Applying (5.1.3) four times, one may write the fourth derivative of $(0, \ldots, 0)$ as a multiple of $(4, \ldots, 4)$. This may then be related to $(5,5,5,5,0)$ by (5.1.2). Applying (5.1.1) to relate $\left(r_{0}, \ldots, r_{4}\right)$ to a linear combination of $\left(r_{0}, \ldots, r_{i}-5, \ldots, r_{4}\right)$ and $\left(r_{0}+1, \ldots, r_{i}-4, \ldots, r_{4}+1\right)$ repeatedly, one can reduce to terms with $r_{i} \leq 4$ for all $i$. In fact, eventually all terms will be of the form $\{(r, r, \ldots, r)\}$ for $r=0, \ldots, 4$. This can be seen by noting that none of (5.1.1), (5.1.1) or (5.1.3) changes $r_{i}-r_{j}(\bmod 5)$. Hence, we have found a relation between the fourth derivative of $(0, \ldots, 0)$ and $\{(r, \ldots, r)\}$ for $r=0, \ldots, 4$. By (5.1.3), the various $(r, \ldots, r)$ are $r$-th derivatives of $(0, \ldots, 0)$, and we obtain a fourth order ODE in $\psi$ for the period corresponding to $P=1$. (See Table 1 below for the equation.) Other cases can be computed similarly. These arguments can be illuminated by diagrams in [4, 12], hence the name diagrammatic technique.

Now we apply this method to calculate the Picard-Fuchs equations for the period integrals we are interested in. For every $g=\left(r_{0}, \ldots, r_{4}\right) \in G$ (defined in Section 2.1), define

$$
P_{g}(x)=x_{0}^{r_{0}} \cdots x_{4}^{r_{4}}
$$

and

$$
k=\left(\sum_{i=0}^{4} \frac{r_{i}}{5}\right)+1=\operatorname{age}(g)+1 .
$$

We will consider specific families of the form

$$
\omega_{g}(\psi)=\operatorname{Res}\left(\frac{\psi P_{g}(x)}{Q_{\psi}(x)^{k}} \Omega_{0}\right)
$$

For our purposes, it will be sufficient to consider families $\omega_{g}$ such that $P_{g}$ satisfies age $(g) \leq 1$ (i.e. $\sum_{i=0}^{4} r_{i} \leq 5$ ) and at least two of the $r_{i}$ 's equal 0 . We observe that other $\omega_{g}$ can be obtained from differentiations (5.1.3) or relations (5.1.1) and (5.1.2) from the listed $\omega_{g}$. For example, $(1,1,1,1,1)$ is the derivative of $(0,0,0,0,0) ;(1,1,1,2,0)$ is related to $(0,0,0,1,4)$ via

$$
0 \equiv x_{3} \partial_{x_{4}} Q_{\psi}=x_{3} x_{4}^{4}-\psi x_{0} x_{1} x_{2} X_{3}^{2} .
$$

We remark that these conditions on $g$ match the conditions on $A$ model computation in Section 3 perfectly. In Claim 6.7 it is shown that the derivatives of these families generate all of $\mathscr{H}$.

Table 1 below gives the Picard-Fuchs equation satisfied by each of the above-mentioned forms. We label the forms by the corresponding 5-tuple $g=\left(r_{0}, \ldots, r_{4}\right)$. Note that permuting the $r_{i}{ }^{\prime}$ s does not effect the differential equation, so we do not distinguish between permutations. Here

$$
t=-5 \log (\psi) \text {. }
$$

The same computation was done in [4, 12]. We note however that there 


\begin{tabular}{|c|c|}
\hline type & Picard-Fuchs equation \\
\hline$(0,0,0,0,0)$ & $\left(\frac{d}{d t}\right)^{4}-5^{5} e^{t}\left(\frac{d}{d t}+\frac{1}{5}\right)\left(\frac{d}{d t}+\frac{2}{5}\right)\left(\frac{d}{d t}+\frac{3}{5}\right)\left(\frac{d}{d t}+\frac{4}{5}\right)$ \\
\hline$(0,0,0,1,4)$ & $\left(\frac{d}{d t}\right)^{2}-5^{5} e^{t}\left(\frac{d}{d t}+2 / 5\right)\left(\frac{d}{d t}+3 / 5\right)$ \\
\hline$(0,0,0,2,3)$ & $\left(\frac{d}{d t}\right)^{2}-5^{5} e^{t}\left(\frac{d}{d t}+1 / 5\right)\left(\frac{d}{d t}+4 / 5\right)$ \\
\hline$(0,0,1,1,3)$ & $\left(\frac{d}{d t}\right)\left(\frac{d}{d t}-1 / 5\right)-5^{5} e^{t}\left(\frac{d}{d t}+1 / 5\right)\left(\frac{d}{d t}+3 / 5\right)$ \\
\hline$(0,0,2,2,1)$ & $\left(\frac{d}{d t}\right)\left(\frac{d}{d t}-2 / 5\right)-5^{5} e^{t}\left(\frac{d}{d t}+1 / 5\right)\left(\frac{d}{d t}+2 / 5\right)$ \\
\hline
\end{tabular}

TABLE 1. The Picard-Fuchs equations for forms $\omega_{g}$.

are several differences between the period integrals we consider, and those of [12]. First, our family $M_{\psi}$ differs from that in [12] by a factor of 5 in the first term. Second, the forms we consider (5.1.4) differ slightly from those considered in [12] by an extra factor of $\psi$ in the numerator (see remark 5.1). Finally, our final equations use different coordinates than in [12]. However the same methods used in their paper can easily be modified to obtain the formulas we present here.

Remark 5.1. The factor of $\psi$ in the numerator of (5.1.4) might appear unnatural at the first glance, but it can be considered as a way to change the form of the Picard-Fuchs equation, as

$$
\frac{d}{d t} e^{-t / 5} f(t)=e^{-t / 5}\left(-\frac{1}{5}+\frac{d}{d t}\right) f(t) .
$$

In the comparison of $A$ model and $B$ model this modification will ensure that the $I$ functions from both sides coincide. It is also used in the Mirror Theorem for the Fermat quintic.

5.2. $I^{B}$-functions. We can solve the above Picard-Fuchs equations with hypergeometric series. As in Section 2, we will organize these solutions in the form of an $I$-function. For each of the above forms $\omega_{g}, I_{g}^{B}$ will be a function taking values in $H_{C R}^{*}(\mathcal{W}) \cong H^{*}(I \mathcal{W})$, whose components give solutions to the corresponding Picard-Fuchs equation. 
Proposition 5.2. For the $g$ listed in table 1 the components of $I_{g}^{B}(t, 1)$ give a basis of solutions to the Picard-Fuchs equations for $\omega_{g}$, where $I_{g}^{B}(t, z)$ is given below.

(i) If $g=e=(0,0,0,0,0)$,

$$
I_{e}^{B}(t, z)=e^{t H / z}\left(1+\sum_{\langle d\rangle=0} e^{d t} \frac{\prod_{\substack{1 \leq m \leq 5 d \\ 0<b \leq d \\\langle b\rangle=0}}(5 H+m z)}{\prod_{0<}(H+b z)^{5}}\right)
$$

(ii) If $g=\left(0,0,0, r_{1}, r_{2}\right)$,

$$
\begin{aligned}
& I_{g}^{B}(t, z)=e^{t H / z} \mathbb{1}_{g} \\
& \left(1+\sum_{\langle d\rangle=0} e^{d t} \frac{\prod_{\substack{0<b \leq d \\
\langle b\rangle=0}}(H+b z)^{3} \prod_{\substack{1 \leq m \leq 5 d \\
0<b \leq d \\
\langle b\rangle=\left\langle\frac{r_{2}}{5}\right\rangle}}(H+b z) \prod_{\substack{0<b \leq d \\
\langle b\rangle=\left\langle\frac{r_{1}}{5}\right\rangle}}(H+b z)}{(H+m z)}\right)
\end{aligned}
$$

(iii) If $g=\left(0,0, r_{1}, r_{1}, r_{2}\right)$, let $g_{1}=\left(-r_{1},-r_{1}, 0,0, r_{2}-r_{1}\right)(\bmod 5)$. Then

$$
\begin{aligned}
& I_{g}^{B}(t, z)=
\end{aligned}
$$

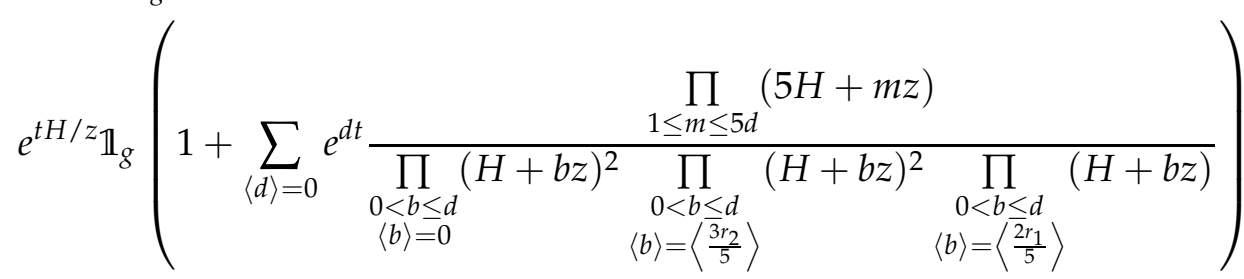

$$
\begin{aligned}
& +e^{t H / z} \mathbb{1}_{g_{1}}\left(\sum_{\langle d\rangle=\left\langle\frac{r_{1}}{5}\right\rangle} e^{d t} \frac{\prod_{\substack{1 \leq m \leq 5 d \\
0<b \leq d \\
\langle b\rangle=\left\langle r_{1}\right.}}(H+b z)^{2} \prod_{\substack{0<b \leq d \\
\langle b\rangle=0}}(H+b z)^{2}}{\prod_{\substack{0<b \leq d \\
\langle b\rangle=\left\langle r_{2} \\
5\right.}}(H+b z)}\right)
\end{aligned}
$$

Remark 5.3. Note that the functions $I_{g}^{B}(t, z)$ in equations (5.2.1), (5.2.2), and (5.2.3), are supported on spaces of dimension 3,1, and 0 respectively. So for each $g$, the number of components of $I_{g}^{B}(t, z)$ equals the order of the corresponding Picard-Fuchs equation as desired.

\section{Mirror THEOREM FOR THE MIRROR QUintiC: $A(\mathcal{W}) \equiv B(M)$}

In this section, we will show the "mirror dual" version of (the mathematical version of) the mirror conjecture by Candelas-de la Ossa-Greene-Parkes [3]. More specifically, we will show that the $A$ model of $\mathcal{W}$ is equivalent to the $B$ model of $M$, up to a mirror map.

We start in 6.1 by stating a "classical" mirror theorem relating the GWT of $\mathcal{W}$ with the periods of $M_{\psi}$ on the level of generating functions. This is 
exactly analogous to Givental's original formulation in [13]. In 6.2 we give a brief explanation of how Givental's original mirror theorem implies a full correspondence between the $A$ model of $M$ and the $B$ model of $\mathcal{W}$. Finally in 6.3 we use similar methods as in 6.2 to prove a mirror theorem equating the $A$ model of $\mathcal{W}$ to the $B$ model of $M$.

6.1. A correspondence of generating functions. We will first show that the $I$-functions $I_{g}^{A}$ of the $A$ model of $\mathcal{W}$ (Definition 3.4) are identical to the $I$-functions $I_{g}^{B}$ of the $B$ model of $M_{\psi}$ defined in Section 5.2

Remark 6.1. Note that in the formula $I_{g}^{A}$, the Novikov variable $q$ always appears next to $e^{t}$. There is therefore no harm in setting $q=1$. We apply this specialization in what follows.

Proposition 6.2. Let $g=\left(r_{0}, \ldots, r_{4}\right) \in G$ satisfies the conditions age $(g) \leq 1$ and that at least two of $r_{i}$ 's are equal to zero. We have an A-interpretation of $g$ as parameterizing a component of $\mathcal{W}_{g}$ in $I \mathcal{W}$. We have also a B-interpretation of $g$ in $\omega_{g}(5.1 .4)$ where $P_{g}$ denote the polynomial $x_{0}^{r_{0}} \cdots x_{4}^{r_{4}}$. Then

$$
I_{g}^{A}(t, z)=I_{g}^{B}(t, z) \text {. }
$$

Proof. This follows from a direct comparison of formulas (3.3.3), (3.3.4), and (3.3.5) from Corollary 3.8 with formulas (5.2.1), (5.2.2), and (5.2.3) respectively.

Combining Proposition 6.2 with Theorem 3.5, we conclude that some periods from VHS of $M$ correspond to the Gromov-Witten invariants of $\mathcal{W}$.

Corollary 6.3. For $g=\left(r_{0}, \ldots, r_{4}\right) \in G$ such that age $(g) \leq 1$ and $\mathcal{W}_{g}$ is nonempty (i.e. at least two $r_{i}$ 's vanish), we have

$$
J_{g}^{\mathcal{W}}(\tau(t), z)=\frac{I_{g}^{B}(t, z)}{H_{g}(t)} \quad \text { where } \tau(t)=\frac{G_{0}(t)}{F_{0}(t)} \text {. }
$$

In other words, under the mirror map

$$
t \mapsto \tau=\frac{G_{0}(t)}{F_{0}(t)},
$$

the periods of $\frac{\omega_{g}}{H_{g}(t)}$ are equal to the coefficients of $J_{g}^{\mathcal{W}}(\tau, 1)$.

This theorem should be viewed as an analogue of Givental's original mirror theorem 6.4 stated below.

6.2. Mirror Theorem for the Fermat quintic revisited. To get some insight of the full correspondence, we return to the "classical" mirror theorem for the Fermat quintic threefold. While this is not strictly necessary for the logical flow of the proof, we feel that it illuminates our approach in a simpler setting. We also strive to clarify certain points which are not entirely clear in the literature. 
Let $J^{M}(t, z)$ denote the small $J$-function for $M$ where $t$ is the coordinate of $H^{2}(M)$ dual to the hyperplane class $H$. Let $\mathcal{W}_{\psi}$ denote the one dimensional deformation family defined by the vanishing of $Q_{\psi}$ (see (5.0.4) ) in $\mathcal{Y}$.

$$
\mathcal{W}_{\psi}:=\left\{Q_{\psi}(x)=0\right\} \subset \mathcal{Y} \text {. }
$$

Let

$$
\omega=\operatorname{Res}\left(\frac{\psi \Omega_{0}}{Q_{\psi}(x)}\right) .
$$

As in section 5 there exists an $H^{*}(M)$-valued $I$-function, $I_{\mathcal{W}_{\psi}}^{B}(t, z)$, such that the components of $I_{\mathcal{W}_{\psi}}^{B}(t, 1)$ give a basis of solutions for the Picard-Fuchs equations for $\omega_{\psi}$, where $t=-5 \log \psi$.

Theorem 6.4 (Mirror Theorem [13][17]). There exist explicitly determined functions $F(t)$ and $G(t)$, such that $F$ is invertible, and

$$
J^{M}(\tau(t), z)=\frac{I_{\mathcal{W}_{\psi}}^{B}(t, z)}{F(t)} \quad \text { where } \tau(t)=\frac{G(t)}{F(t)} .
$$

We will show how Theorem 6.4 implies a correspondence between the fundamental solution matrix of the Dubrovin connection for $M$ and that of the Gauss-Manin connection for $\mathcal{W}_{\psi}$. In order to emphasize the symmetry between the $A$ model and $B$ model, we will denote the respective pairings as $(-,-)^{A}$ and $(-,-)^{B}$.

Let

$$
s=e^{t}=\psi^{-5},
$$

and consider the flat family $\mathcal{W}_{s}$ over $S=\operatorname{Spec}(\mathbb{C}[s])$. In the Calabi-Yau case, the $H$ expansion of $I^{B}$ always occurs in the form of a function of $H / z$, in particular $I_{\mathcal{W}_{s}}^{B}$ is homogeneous of degree zero if one sets $\operatorname{deg}(z)=2$. The same is true of $J^{M}$. Thus, one may set $z=1$ without loss of information. $I_{\mathcal{W}_{s}}^{B}(t, 1)$ gives a basis of solutions for the Picard-Fuchs equations of $\omega$. In other words after an appropriate choice of basis $\left\{s_{0}^{B}(t), \ldots, s_{3}^{B}(t)\right\}$ of solutions of $\nabla^{G M}$,

$$
\left(s_{i}^{B}(t), \omega\right)^{B}=I_{i}^{B}(t, 1),
$$

where $I_{i}^{B}(t, z)$ is the $H^{i}$ coefficient of $I_{\mathcal{W}_{s}}^{B}(t, z)$.

By the same argument, if we choose an appropriate basis $\left\{s_{0}^{A}(\tau), \ldots, s_{3}^{A}(\tau)\right\}$ of solutions for $\nabla^{z}$, Section 1 shows that the coefficients $J_{i}^{M}(\tau, 1)$ of the function $J^{M}(\tau, 1)$ give us the functions

$$
\left(s_{i}^{A}(\tau), 1\right)^{A}=J_{i}^{M}(\tau, 1) .
$$

Thus we can interpret Theorem 6.4 as saying that after choosing correct bases of flat sections and applying the mirror map

$$
t \mapsto \tau=\frac{G(t)}{F(t)}
$$


we have the equality

$$
\left(s_{i}^{B}(t), \omega / F(t)\right)^{B}=\frac{I_{i}(t, 1)}{F(t)}=J_{i}(\tau, 1)=\left(s_{i}^{A}(\tau), 1\right)^{A} .
$$

To show the full correspondence between the solution matrix for the Dubrovin connection for $M$ and that of the Gauss-Manin connection on $S$, we must find a basis $\phi_{0}, \ldots, \phi_{3}$ of sections of $\mathscr{H}$ and a basis $T_{0}, \ldots, T_{3}$ of sections of $H^{\text {even }}(M)$ such that for all $i$ and $j$,

$$
\left(s_{i}^{B}, \phi_{j}\right)^{B}=\left(s_{i}^{A}, T_{j}\right)^{A}
$$

As expected, we set $\phi_{0}=\omega / F(t)$ and $T_{0}=1$.

\section{Claim 6.5.}

$$
\phi_{j}=\left(\nabla_{t}^{G M}\right)^{j} \phi_{0} \text { for } 0 \leq j \leq 3
$$

gives a basis of sections for $\mathscr{H}$.

Proof. This follows from standard Hodge theory for Calabi-Yau threefolds, but in this case can be explicitly calculated.

$$
\begin{aligned}
\nabla_{t}^{G M} \phi_{0} & =\frac{d}{d t}\left(\frac{1}{F(t)}\right) \omega+\frac{1}{F(t)} \nabla_{t}^{G M} \omega \\
& =-\frac{F^{\prime}(t)}{F(t)} \phi_{0}+\frac{1}{F(t)} \operatorname{Res}\left(\frac{d}{d t} \frac{\psi \Omega_{0}}{Q_{\psi}}\right) \\
& =-\frac{F^{\prime}(t)}{F(t)} \phi_{0}+\frac{1}{F(t)} \operatorname{Res}\left(s \frac{d}{d s} \frac{\psi \Omega_{0}}{Q_{\psi}}\right) \\
& =-\frac{F^{\prime}(t)}{F(t)} \phi_{0}+\frac{1}{F(t)} \operatorname{Res}\left(\frac{-\psi}{5} \frac{d}{d \psi} \frac{\psi \Omega_{0}}{Q_{\psi}}\right) \\
& =-\frac{F^{\prime}(t)}{F(t)} \phi_{0}+\frac{-\psi}{5 F(t)} \operatorname{Res}\left(\frac{\Omega_{0}}{Q_{\psi}}+\frac{x_{0} \cdots x_{4}}{Q_{\psi}^{2}} \Omega_{0}\right)
\end{aligned}
$$

Because of the last term in the above sum, the image of $\left(\nabla_{t}^{G M}\right) \phi_{0}$ in $\mathscr{F}^{2} / \mathscr{F}^{3}$ is nonzero by (4.2.2). Similarly, the image of $\left(\nabla_{t}^{G M}\right)^{j} \phi_{0}$ in $\mathscr{F}^{3-j} / \mathscr{F}^{3+1-j}$ for $1 \leq j \leq 3$ is nonzero, thus the sections $\phi_{0}, \ldots, \phi_{3}$ must be linearly independent.

Note that

$$
\begin{aligned}
& \left(s_{i}^{B}, \phi_{1}\right)^{B}=\left(s_{i}^{B}, \nabla_{t}^{G M} \phi_{0}\right)^{B}=\frac{\partial}{\partial t}\left(s_{i}^{B}, \phi_{0}\right)^{B}= \\
& \frac{\partial}{\partial t}\left(s_{i}^{A}, T_{0}\right)^{A}=\left(\frac{\partial \tau}{\partial t}\right) \frac{\partial}{\partial \tau}\left(s_{i}^{A}, T_{0}\right)^{A}=\left(s_{i}^{A},\left(\frac{\partial \tau}{\partial t}\right) \nabla_{\tau}^{z} T_{0}\right)^{A} .
\end{aligned}
$$

Therefore, if we set

$$
T_{1}=\frac{\partial(G / F)}{\partial t} \nabla_{\tau}^{z} T_{0}
$$


we have the desired relationship

$$
\left(s_{i}^{B}, \phi_{1}\right)^{B}=\left(s_{i}^{A}, T_{1}\right)^{A} .
$$

If we similarly set

$$
T_{k}=\frac{\partial(G / F)}{\partial t} \nabla_{\tau}^{z} T_{k-1}
$$

6.2.2) follows.

This shows that the mirror map lifts to an isomorphism of vector bundles, and the connection is preserved. Indeed, the fundamental solution of the Gauss-Manin connection is a 4 by 4 matrix, where 4 is the rank of $H^{3}(\mathcal{W})$. On the other hand, the fundamental solution of the Dubrovin connection is also a 4 by 4 matrix, where 4 is the rank of $H^{e v e n}(M)$. We recall that the $J$-function can be thought of as the first row vectors of the fundamental solution matrix, as discussed in Section 1. The above discussion shows that we can extend the correspondence between the first row of the fundamental solution to the full fundamental solution.

We summarize the above in the following theorem.

Theorem 6.6. The fundamental solutions of the Gauss-Manin connection for $\mathcal{W}_{s}$ are equivalent, up to a mirror map, to the fundamental solutions of the Dubrovin connection for $M$, when restricted to $H^{2}(M)$.

6.3. Mirror Theorem for the mirror quintic. In this subsection, we will extend the partial correspondence in Section 6.1 between the periods of $M_{\psi}$ and the $A$ model of $\mathcal{W}$ to the full correspondence, generalizing the ideas in Section 6.2.

Similar to the above, consider the flat family $M_{s}$ over $S=\operatorname{Spec}(\mathbb{C}[s])$ defined by (5.0.4), where $s=e^{t}=\psi^{-5}$. Corollary 6.3 states that some periods of $M_{s}$ correspond to Gromov-Witten invariants on $\mathcal{W}$. We would like to extend this result to all periods.

First, we must choose a basis of sections of $\mathscr{H} \rightarrow S$. Let $\omega_{e}$ denote the holomorphic family of $(3,0)$-forms corresponding to $g=e=(0, \ldots, 0)$ in (5.1.4). It is no longer true that derivatives of $\omega_{e} / F_{0}(t)$ with respect to the Gauss-Manin connection generate a basis of sections of $\mathscr{H}$, thus it becomes necessary to consider the other forms $\omega_{g}$ satisfying the conditions formulated in Corollary 6.3. Namely, let $\phi_{e}=\omega / F_{0}(t)$ and let $\phi_{g}=\omega_{g} / H_{g}(t)$ where $g$ satisfies age $(g)=1$. Consider the set of sections

$$
\left\{\phi_{0}, \nabla_{t}^{G M} \phi_{0},\left(\nabla_{t}^{G M}\right)^{2} \phi_{0},\left(\nabla_{t}^{G M}\right)^{3} \phi_{0}\right\} \cup\left\{\phi_{g}, \nabla_{t}^{G M} \phi_{g}\right\} .
$$

Claim 6.7. These forms comprise a basis of the Hodge bundle $\mathscr{H}$.

Proof. The proof is similar to Claim 6.5. We note that in the last four rows in Table 1, corresponding to age one type, the dimensions are 20,20,30, and 30. Thus $\left|\left\{\phi_{g}\right\}\right|=100$, and there are exactly 204 forms in the above set. One can check via (4.2.2) and another argument like in (6.2.3) that these sections are in fact linearly independent. 
Then, as in (6.2.4) the periods of $\left(\nabla_{t}^{G M}\right)^{k} \phi_{0}$ correspond to the derivatives $\left(\frac{d}{d t}\right)^{k} J_{e}^{\mathcal{W}}(\tau, 1)$, and the periods of $\nabla_{t}^{G M} \phi_{g}$ correspond to $\left(\frac{d}{d t}\right) J_{g}^{\mathcal{W}}(\tau, 1)$.

Let $T_{0}=1$, and $T_{k}=\frac{\partial\left(G_{0} / F_{0}\right)}{\partial t} \nabla_{\tau}^{z} T_{k-1}$ for $0 \leq k \leq 3$. Let $T_{g}=\mathbb{1}_{g}$ and $T_{g}^{\prime}=\frac{\partial\left(G_{0} / F_{0}\right)}{\partial t} \nabla_{\tau}^{z} \mathbb{1}_{g}$. Then if we choose the correct basis of flat sections $\left\{s_{i}^{B}\right\}$ and $\left\{s_{i}^{A}\right\}$, we have that

$$
\begin{aligned}
\left(s_{i}^{B},\left(\nabla_{t}^{G M}\right)^{k} \phi_{0}\right)^{B} & =\left(s_{i}^{A}, T_{k}\right)^{A}, \\
\left(s_{i}^{B}, \phi_{g}\right)^{B} & =\left(s_{i}^{A}, T_{g}\right)^{A} \text { and } \\
\left(s_{i}^{B}, \nabla_{t}^{G M} \phi_{g}\right)^{B} & =\left(s_{i}^{A}, T_{g}^{\prime}\right)^{A} .
\end{aligned}
$$

This implies that the set

$$
\left\{T_{0}, T_{1}, T_{2}, T_{3}\right\} \cup\left\{T_{g}, T_{g}^{\prime}\right\},
$$

is a basis of $T H_{C R}^{\text {even }}(\mathcal{W})$, and that with these choices of bases the solution matrices for the two respective connections are identical after the mirror transformation. Thus we obtain the full correspondence.

In terms of the language of Theorem 6.6, we can formulate our final result in the following form. On the side of the $A$ model of $\mathcal{W}$, let $t$ be the dual coordinate of $H$; on the side of $B$ model of $M_{s}$, let $t=\log (s)$. Then we have

Theorem 6.8. The fundamental solutions of the Gauss-Manin connection $\nabla_{t}^{G M}$ for $M_{s}$ is equivalent, up to a mirror map, to the fundamental solutions of the Dubrovin connection $\nabla_{t}^{z}$ for $\mathcal{W}$ restricted to $t H \in H^{2}(\mathcal{W})$.

Remark 6.9. Even though the base direction is constrained to one dimension instead of the full 101-dimension deformation space, our fundamental solutions are full 204 by 204 matrices, as both ranks of $H^{3}(M)$ and $H^{\text {even }}(\mathcal{W})$ are 204.

\section{REFERENCES}

[1] D. Abramovich, T. Graber, A. Vistoli, Gromov-Witten theory of Deligne-Mumford stacks, Amer. J. Math., 130 (2008), 1337-1398.

[2] R. Bryant and P. Griffiths, Some observations on the infinitesimal period relations for regular threefolds with trivial canonical bundle, Arithmetic and geometry, Vol. II, 77-102, Progr. Math., 36, Birkhäuser Boston, Boston, MA, 1983.

[3] P. Candelas, X. De La Ossa, P. Green, and L. Parkes, A pair of Calabi-Yau manifolds as an exactly soluble superconformal field theory, Nuclear Phys. B 359 (1991), 21-74.

[4] P. Candelas, X. de la Ossa, F. Rodriguez-Villegas, Calabi-Yau Manifolds Over Finite Fields, I, http://arxiv.org/abs/hep-th/0012233.

[5] W. Chen and Y. Ruan, A new cohomology theory of orbifold, Comm. Math. Phys., 248 (2004), 1-31.

[6] W. Chen and Y. Ruan, Orbifold Gromov-Witten theory, in Orbifolds in mathematics and physics (Madison, WI, 2001), pp. 25-85. Contemp Math., 310. Amer. Math. Soc., Providence, RI, 2002.

[7] T. Coates, A. Corti, H. Iritani, H.-H. Tseng, Computing genus-zero twisted Gromov-Witten invariants, Duke Math. J., 147 (2009), no. 3, 377-438. 
[8] T. Coates, A. Corti, Y.-P. Lee, H.-H. Tseng, The quantum orbifold cohomology of weighted projective spaces, Acta Math. 202 (2009), no. 2, 139-193.

[9] D. A. Cox and S. Katz; Mirror Symmetry and Algebraic Geometry, Math. Surv. Mono. 68, Amer. Math. Soc. 1999.

[10] D. A. Cox, S. Katz and Y.-P. Lee; Virtual fundamental classes of zero loci, Advances in algebraic geometry motivated by physics (Lowell, MA, 2000), 157-166, Contemp. Math., 276, Amer. Math. Soc., Providence, RI, 2001.

[11] P. Deligne, Equations différentielles à points singuliers réguliers, Lecture Notes in Mathematics, Vol. 163. Springer-Verlag, Berlin-New York, 1970.

[12] C. Doran, B. Greene, S. Judes, Families of quintic Calabi-Yau 3-folds with discrete symmetries, Comm. Math. Phys., 280 (2008), 675-725.

[13] A. B. Givental, Equivariant Gromov-Witten invariants, Internat. Math. Res. Notices 1996, no. 13, 613-663.

[14] A. B. Givental, A mirror theorem for toric complete intersections, in Topological field theory, primitive forms and related topics (Kyoto, 1996), pp. 141-175. Progr. Math., 160. Birkhäuger Boston, Boston, MA, 1998.

[15] P. A. Griffiths, On the periods of certain rational integrals, Ann. of Math., (2) 90 (1969) 460-495.

[16] P. Griffiths, ed., Topics in transcendental algebraic geometry, Annals of Mathematics Studies, 106. Princeton University Press, Princeton, NJ, 1984.

[17] B. H. Lian, K. Liu, and S.-T. Yau, Mirror Principle. I, Asian J. Math., 1 (1997), 729-763.

[18] T. Graber and R. Pandharipande, Localization of virtual classes, Invent. Math., 135 (1999), 487-518.

[19] B. Greene and M. R. Plesser, Duality in Calabi-Yau moduli space, Nuclear Phys. B 338 (1990), no. 1, 15-37.

[20] E. Witten, Mirror manifolds and topological field theory, Essays on mirror manifolds, 120158, Int. Press, Hong Kong, 1992.

Department of Mathematics, University of Utah, SAlt LAKe City, Utah 84112 0090, U.S.A.

E-mail address: yplee@math.utah.edu

Department of Mathematics, University of Michigan, AnN Arbor, Mi 481091043 , U.S.A.

E-mail address: shoemama@umich.edu 